\title{
Non-homogeneous polygonal Markov fields in the plane: graphical representations and geometry of higher order correlations
}

\author{
Tomasz Schreiber* \\ Faculty of Mathematics \& Computer Science, \\ Nicolaus Copernicus University, Toruń, Poland.
}

\begin{abstract}
We consider polygonal Markov fields originally introduced by Arak and Surgailis [2]. Our attention is focused on fields with nodes of order two, which can be regarded as continuum ensembles of non-intersecting contours in the plane, sharing a number of features with the two-dimensional Ising model. We introduce non-homogeneous version of polygonal fields in anisotropic enviroment. For these fields we provide a class of new graphical constructions and random dynamics. These include a generalised dynamic representation, generalised and defective disagreement loop dynamics as well as a generalised contour birth and death dynamics. Next, we use these constructions as tools to obtain new exact results on the geometry of higher order correlations of polygonal Markov fields in their consistent regime.
\end{abstract}

Keywords Arak-Surgailis polygonal Markov fields, graphical construction, dynamic representation, higher order correlation functions, two-dimensional Ising model

\section{Introduction}

The first example of a polygonal Markov field has been provided by Arak [1]. Further developments are due to Arak \& Surgailis [2, 3], Surgailis [18] and Arak, Clifford \& Surgailis [4]. The fields with nodes of order 2, or V-shaped nodes for short, which are in the focus of

${ }^{*}$ Mailing address: Tomasz Schreiber, Faculty of Mathematics \& Computer Science, Nicolaus Copernicus University, ul. Chopina 12 / 18, 87-100 Toruń, Poland; tel.: $(++48)(+56) 6112951$, fax: $(++48)(+56)$ 6228979; e-mail: tomeks at mat.uni.torun.pl 
our attention in this paper, arise as ensembles of self-avoiding closed polygonal contours in the plane interacting by hard core exclusions, possibly with some further terms entering the Hamiltonian. Under a particular choice of the Hamiltonian the polygonal fields enjoy striking properties including consistency (the field constructed in a subdomain $D \subseteq D^{\prime} \subseteq \mathbb{R}^{2}$ coincides with the restriction to $D$ of the field constructed in $D^{\prime}$ ) as well as availability of an explicit formula for the partition function and some other numerical characteristics of the field, see [2]. Under even milder conditions one can guarantee the isometry invariance of the field as well as the two-dimensional germ-Markov property stating that the conditional behaviour of the field in an open bounded domain depends on the exterior configuration only through arbitrarily close neighbourhoods of the boundary, see ibidem. A particularly interesting class of processes seem to be length- and area-interacting modifications of consistent fields, which not unexpectedly exhibit many features analogous to those of the two-dimensional Ising model, including the presence of first order phase transition at low enough temperatures (Nicholls, [14]; Schreiber, [15]) and low-temperature phase separation phenomenon (Schreiber, [16]). Consistent polygonal fields and their length-interacting modifications have also interesting statistical applications where they are used as priors in Bayesian image analysis (Clifford \& Nicholls, [6]; Kluszczyński, van Lieshout \& Schreiber, $[10,11]$; van Lieshout \& Schreiber, [12, 17]).

With all the afore-mentioned similarities the polygonal fields enjoy with the Ising model as well as with more general lattice models used in Bayesian image analysis, one salient difference is that whereas the latter can be analysed using a rich mixture of combinatorial, analytic and geometric techniques, the overwhelming majority of crucial results for the polygonal Markov fields have been obtained via purely geometric methods usually going under the guise of graphical constructions. These were first provided by Arak \& Surgailis $[2,3]$ and Arak, Clifford \& Surgailis [4] in the form of the so-called dynamic representations for consistent polygonal fields. Later, we have introduced disagreement loop dynamics and contour birth and death representation for broader classes of polygonal fields, see [15]. The purpose of the present paper is twofold.

- First, we introduce a class of new graphical constructions and random dynamics for a rather general class of non-homogeneous polygonal Markov fields with V-shaped nodes. These constructions include a generalised dynamic representation, generalised and defective disagreement loop dynamics as well as a generalised contour birth and death dynamics. 
- Next, we use these constructions as tools to obtain new exact results on the geometry of higher order correlations of polygonal Markov fields in the consistent regime.

We envision to use the new graphical representations in our current research in progress on polygonal fields, especially in context of obtaining further exact formulae for higher order correlations and tentatively also to obtain exact formulae for the free energy of the field (exact solution) at least in the rectangular case. Due to their intrinsically algorithmic nature the new constructions will also be used for Bayesian image segmentation purposes, which is our work in progress in cooperation with van Lieshout, see Schreiber \& van Lieshout [17] for some partial developments in the particular tesselation-based set-up.

The paper is organised as follows. In Section 2 below we introduce general nonhomogeneous polygonal fields in non-homogeneous and anisotropic environments. This is done by imposing a general activity measure on the space of straight lines in the plane, determining how likely the presence of an edge along a given line is. The usual Ising-type length interaction is replaced by its anisotropic version there - the energy cost of producing an edge crossing lines of high activities is higher than the cost of an edge of the same length crossing only low activity lines. Further, in Section 3 general properties of such fields in their consistent regime are discussed and a non-homogeneous counterpart of the Arak \& Surgailis [2] dynamic representation is developed. Next, in Section 4 we develop a generalised dynamic representation for consistent polygonal fields, which allows for a flexible choice of the field creation dynamics, parametrised by an increasing family of convex compact subsets of the field domain. In the subsequent Section 5 we employ the generalised dynamic representation to provide exact factorisation statements for higher order correlation functions of polygonal fields in consistent regime. In the next Section 6 we introduce variants of the so-called disagreement loop dynamics for polygonal fields, including the generalised and defective disagreement loop dynamics. These are then used in Section 7 to establish exponential decay of higher order correlations in the particular set-up of rectangular fields. Finally, in Section 8 we introduce general contour birth and death dynamics for polygonal fields in low enough temperature, in the spirit of Fernández, Ferrari \& Garcia $[7,8,9]$ and yielding a perfect simulation scheme directly from the thermodynamic limit. 


\section{Non-homogeneous polygonal Markov fields}

For an open bounded convex set $D$ define the family $\Gamma_{D}$ of admissible polygonal configurations on $D$ by taking all the finite planar graphs $\gamma$ in $D \cup \partial D$, with straight-line segments as edges, such that

(P1) the edges of $\gamma$ do not intersect,

(P2) all the interior vertices of $\gamma$ (lying in $D$ ) are of degree 2,

(P3) all the boundary vertices of $\gamma$ (lying in $\partial D$ ) are of degree 1 ,

(P4) no two edges of $\gamma$ are colinear.

In other words, $\gamma$ consists of a finite number of disjoint polygons, possibly nested and chopped off by the boundary. Further, for a finite collection $(l)=\left(l_{i}\right)_{i=1}^{n}$ of straight lines intersecting $D$ we write $\Gamma_{D}(l)$ to denote the family of admissible configurations $\gamma$ with the additional properties that $\gamma \subseteq \bigcup_{i=1}^{n} l_{i}$ and $\gamma \cap l_{i}$ is a single interval of a strictly positive length for each $l_{i}, i=1, \ldots, n$, possibly with some isolated points added.

We shall also consider the subfamily $\Gamma_{D \mid \emptyset} \subseteq \Gamma_{D}$ of empty boundary polygonal configurations in $D$ consisting of those $\gamma \in \Gamma_{D}$ which have no boundary vertices. For $(l)$ as above we put $\Gamma_{D \mid \emptyset}(l):=\Gamma_{D}(l) \cap \Gamma_{D \mid \emptyset}$.

For a Borel subset of $A \subseteq \mathbb{R}^{2}$ by $[[A]]$ we shall denote the family of all straight lines hitting $A$ so that in particular $\left[\left[\mathbb{R}^{2}\right]\right]$ stands for the collection of all straight lines in $\mathbb{R}^{2}$. We shall also write $\overline{[[A]]}$ for the family of all linear segments in $\mathbb{R}^{2}$ hitting $A$. Consider a non-negative Borel measure $\mathcal{M}$ on the $\left[\left[\mathbb{R}^{2}\right]\right]$ such that

(M1) $\mathcal{M}([[A]])<\infty$ for all bounded Borel $A \subseteq \mathbb{R}^{2}$,

(M2) $\mathcal{M}([[\{x\}]])=0$ for all $x \in \mathbb{R}^{2}$.

Below, the measure $\mathcal{M}$ will be interpreted as the activity measure on $\left[\left[\mathbb{R}^{2}\right]\right]$. Let $\Lambda^{\mathcal{M}}$ be the Poisson line process on $\left[\left[\mathbb{R}^{2}\right]\right]$ with intensity measure $\mathcal{M}$ and write $\Lambda_{D}^{\mathcal{M}}$ for its restriction to the domain $D$. Further, define the Hamiltonian $L^{\mathcal{M}}: \Gamma_{D} \rightarrow \mathbb{R}_{+}$given by

$$
L^{\mathcal{M}}(\gamma):=\sum_{e \in \operatorname{Edges}(\gamma)} \mathcal{M}([[e]]), \quad \gamma \in \Gamma_{D}
$$


We argue that the energy function $L^{\mathcal{M}}$ should be regarded as an anisotropic environmentspecific version of the length functional. Indeed, interpreting the activity $\mathcal{M}(d l)$ of a line $l$ hitting an edge $e \in \operatorname{Edges}(\gamma)$ as the likelihood of a new edge being created along $l$ intersecting and hence fracturing the edge $e$ in $\gamma$, we note that, roughly speaking, the value of $\mathcal{M}([[e]])$ determines how likely the edge $e$ is to be fractured by another edge present in the environment. In other words, $L^{\mathcal{M}}(\gamma)$ determines how difficult it is to create the whole graph $\gamma \in \Gamma_{D}$ without fractures in the environment $\mathcal{M}$ - note that due to the anisotropy of the environment there may be graphs of a higher (lower) total edge length than $\gamma$ and yet of lower (higher) energy and thus easier (more difficult) to create and to keep unfractured due to the lack (presence) of high activity lines likely to fracture their edges.

With the above notation, for $\beta \in \mathbb{R}$ further referred to as the inverse temperature (for mathematical convenience we admit also the unphysical negative values of inverse temperatures), we define the polygonal field $\mathcal{A}_{D}^{\mathcal{M} ; \beta}$ in $D$ with activity measure $\mathcal{M}$ to be the Gibbsian modification of the process induced on $\Gamma_{D}$ by $\Lambda_{D}^{\mathcal{M}}$, with the Hamiltonian $L^{\mathcal{M}}$ at inverse temperature $\beta$, that is to say

$$
\mathbb{P}\left(\mathcal{A}_{D}^{\mathcal{M} ; \beta} \in G\right):=\frac{\mathbb{E} \sum_{\gamma \in \Gamma_{D}\left(\Lambda_{D}^{\mathcal{M}}\right) \cap G} \exp \left(-\beta L^{\mathcal{M}}(\gamma)\right)}{\mathbb{E} \sum_{\gamma \in \Gamma_{D}\left(\Lambda_{D}^{\mathcal{M}}\right)} \exp \left(-\beta L^{\mathcal{M}}(\gamma)\right)}
$$

for all sets $G \subseteq \Gamma_{D}$ Borel measurable with respect to, say, the usual Hausdorff distance topology. Note that this definition can be rewritten as

$$
\mathbb{P}\left(\mathcal{A}_{D}^{\mathcal{M} ; \beta} \in d \gamma\right) \propto \exp \left(-\beta L^{\mathcal{M}}(\gamma)\right) \prod_{e \in \operatorname{Edges}(\gamma)} \mathcal{M}(d l[e]), \gamma \in \Gamma_{D}
$$

where $l[e]$ is the straight line extending $e$. In other words, the probability of having $\mathcal{A}_{D}^{\mathcal{M} ; \beta} \in$ $d \gamma$ is proportional to the Boltzmann factor $\exp \left(-\beta L^{\mathcal{M}}(\gamma)\right)$ times the product of edge activities $\mathcal{M}(d l[e]), e \in \operatorname{Edges}(\gamma)$. Observe also that this construction should be regarded as a specific version of the general polygonal model given in (2.11) in [2]. The finiteness of the partition function

$$
\mathcal{Z}_{D}^{\mathcal{M} ; \beta}:=\mathbb{E} \sum_{\gamma \in \Gamma_{D}\left(\Lambda_{D}^{\mathcal{M}}\right)} \exp \left(-\beta L^{\mathcal{M}}(\gamma)\right)<\infty
$$

for all $\beta \in \mathbb{R}$ is not difficult to verify, see (32) below. We shall also consider the empty boundary version of the above construction, with $\mathcal{A}_{D \mid \emptyset}^{\mathcal{M} ; \beta}$ arising in law as $\mathcal{A}_{D}^{\mathcal{M} ; \beta}$ conditioned on staying within $\Gamma_{D \mid \emptyset}$, that is to say not containing boundary vertices. It is easily seen 
that alternatively $\mathcal{A}_{D \mid \emptyset}^{\mathcal{M} ; \beta}$ can be defined by rewriting (2) above with $\Gamma_{D}$ replaced with $\Gamma_{D \mid \emptyset}$ throughout. The corresponding partition function $\mathcal{Z}_{D \mid \phi}^{\mathcal{M} ; \beta}$ is always finite as well, see (31) below.

In the sequel we also consider the thermodynamic limits for such fields, which take their values in the space $\Gamma:=\Gamma_{\mathbb{R}^{2}}$ of whole-plane admissible configurations, with obvious meaning of this notation. Note in this context that $\Gamma_{\mathbb{R}^{2}}=\Gamma_{\mathbb{R}^{2} \mid \emptyset \text {. }}$

As a particular case of this general construction we shall consider rectangular fields. Slightly abusing the terminology, by a rectangular field we shall mean a polygonal field given by (2) and (3) whose activity measure $\mathcal{M}$ concentrates on translates of two nonparallel straight lines - in other words, its edges can follow precisely two different directions. Clearly, upon a non-singular affine transform such a rectangular field can be made into a process where all angles between edges are straight, whence the name. Assuming with no loss of generality that the two admissible directions for the edges of the field are parallel to the coordinate axes, we can further transform each rectangular field into the standard rectangular field where the measure $\mathcal{M}$ is given by $\mathcal{M}(d l)$ being $d h$ if $l$ is a parallel translate of a coordinate axe by distance $h, h \in \mathbb{R}$, and 0 otherwise. Indeed, putting

$$
F_{\leftrightarrow}(x):=\left\{\begin{array}{cl}
\mathcal{M}((0, x] \times\{0\}), & \text { if } x \geq 0, \\
-\mathcal{M}((x, 0] \times\{0\}), & \text { otherwise }
\end{array}\right.
$$

and

$$
F_{\uparrow}(y):=\left\{\begin{array}{cl}
\mathcal{M}(\{0\} \times(0, y]), & \text { if } y \geq 0, \\
-\mathcal{M}(\{0\} \times(y, 0]), & \text { otherwise, }
\end{array}\right.
$$

it is readily verified that the map $\mathbb{R}^{2} \ni(x, y) \mapsto\left(F_{\leftrightarrow}(x), F_{\uparrow}(y)\right)$ does define such a transformation. Note that both $F_{\leftrightarrow}$ and $F_{\uparrow}$ are finite by (M1) and continuous by (M2). Although in general this transformation is not a bijection, it is easily seen to be bijective on the support of $\mathcal{A}^{\mathcal{M}}$, with the inverse defined by $(s, t) \mapsto\left(\sup \left\{x, F_{\leftrightarrow}(x) \leq s\right\}, \sup \left\{y, F_{\uparrow}(y) \leq t\right\}\right)$.

Even though in general we admit all $\beta \in \mathbb{R}$, in this paper we shall mainly study two principal temperature regions. The first one is the consistent regime corresponding to $\beta=1$ and introduced in Section 3 - we shall argue that this particular choice of temperature parameter places us in the context of a non-homogeneous version of Arak-Surgailis [2] construction for the so-called consistent polygonal fields, thus ensuring the availability of an appropriate dynamic representation for our process in terms of equilibrium evolution of one-dimensional particle systems tracing the boundaries of the field in two-dimensional space-time, as discussed in detail in Subsection 3.1 below, see also [2]. The afore-mentioned 
consistency property, arising as a consequence of the dynamic representation and further discussed in Subsection 3.2, means here that for each open bounded convex $D \subseteq D^{\prime} \subset \mathbb{R}^{2}$ the field $\mathcal{A}^{\mathcal{M} ; 1}:=\mathcal{A}_{D}^{\mathcal{M} ; 1}$ coincides in law with $\mathcal{A}_{D^{\prime}}^{\mathcal{M}}:=\mathcal{A}_{D^{\prime}}^{\mathcal{M} ; 1} \cap D$, thus allowing for a direct construction of the infinite volume process (thermodynamic limit) $\mathcal{A}^{\mathcal{M}}:=\mathcal{A}^{\mathcal{M} ; 1}$ on the whole $\mathbb{R}^{2}$. A number of further properties can be concluded from the dynamic representation for $\beta=1$, including the explicit knowledge of the partition function $Z_{D}^{\mathcal{M} ; 1}$ as made precise in Theorem 2 below. The consistent fields are the main object of study in Sections 3, 4, 5 and 7, and play a crucial role also in Section 6 .

The second regime in the focus of our interest is the low temperature region (large positive $\beta$ ) where long range point-to-point correlations are present, giving rise to the spontaneous magnetisation phenomenon, see Corollary 6 in Section 8 and the discussion below it. All disagreement loop dynamics developed in Section 6 of this paper admit their versions working for the low temperature region. Moreover, in Section 8 we provide another important tool characterising the behaviour of our model in this regime, namely a contour birth and death graphical construction in the spirit of Fernández, Ferrari and Garcia [7, 8, 9] put together with our random walk representation for the so-called free contour measure specific to the model. Applying this graphical construction we are able to establish the existence of the thermodynamic limit for $\mathcal{A}^{\mathcal{M} ; \beta}$ as well as certain mixing-type results for general activity measures satisfying (27) in analogy with Section 4 in [15], see Corollary 5.

As a by-product of our considerations both in the consistent and low temperature regimes we obtain efficient exact simulation algorithms for $\mathcal{A}^{\mathcal{M} ; \beta}$, both in finite windows and directly from the thermodynamic limit.

\section{The consistent regime}

As mentioned above, we show here that the choice $\beta=1$ puts us in the so-called consistent regime of the polygonal field, where it enjoys remarkable features arising as consequences of a (non-homogeneous) version of the dynamic representation developed by Arak \& Surgailis [2], see Section 4 there. In Subsection 3.1 below, we give the non-homogeneous dynamic representation and further in Subsection 3.2 we discuss its consequences. 


\subsection{Consistent fields and their dynamic representation}

To describe the dynamic representation, we interpret the open convex domain $D$ as a set of space-time points $(t, y) \in D$, with $t$ referred to as the time coordinate and with $y$ standing for the spatial coordinate of a particle at the time $t$. In this language, a straight line segment in $D$ stands for a piece of the space-time trajectory of a freely moving particle. In the sequel by a particle we shall understand a function $t \mapsto y_{t}$ assigning to each time moment $t$ the spatial location $y_{t}$ of the particle at the time $t$. Moreover, by the trajectory of a particle we shall mean the graph of this function, i.e. the set of points $\left(t, y_{t}\right)$. The particle trajectories considered in this paper are piecewise linear and hence almost everywhere differentiable. Clearly, in the language being introduced, the derivative of $y_{t}$ at $t$ corresponds to the current velocity of the particle at the time $t$. Below, for notational convenience we shall omit the subscript $t$ writing $y$ instead of $y_{t}$. For a straight line $l$ non-parallel to the spatial axis and crossing the domain $D$ we define in the obvious way its entry point to $D, \operatorname{in}(l, D) \in \partial D$ and its exit point $\operatorname{out}(l, D) \in \partial D$.

On $\mathbb{R}^{2}$ we construct the birth measure $\langle\langle\mathcal{M}\rangle\rangle$ given for each Borel $A \subseteq \mathbb{R}^{2}$ by

$$
\langle\langle\mathcal{M}\rangle\rangle(A):=\frac{1}{2} \mathcal{M} \times \mathcal{M}\left(\left\{\left(l_{1}, l_{2}\right), l_{1} \cap l_{2} \subset A\right\}\right)=\mathbb{E} \operatorname{card}\left\{\left\{l_{1}, l_{2}\right\} \subseteq \Lambda^{\mathcal{M}}, l_{1} \neq l_{2}, l_{1} \cap l_{2} \subset A\right\} .
$$

Note in this context that $\mathcal{M} \times \mathcal{M}\left(\left\{(l, l), l \in\left[\left[\mathbb{R}^{2}\right]\right]\right\}\right)=0$ by (M2). Likewise, on $\partial D$ we construct the boundary birth measure $\langle\langle\mathcal{M} ; \partial D\rangle\rangle$ given for each Borel $B \subseteq \partial D$ by

$$
\langle\langle\mathcal{M} ; \partial D\rangle\rangle(B):=\mathcal{M}(\{l, \operatorname{in}(l, D) \in B\})=\mathbb{E} \operatorname{card}\left\{l \in \Lambda^{\mathcal{M}}, \operatorname{in}(l, D) \in B\right\} .
$$

We choose the space-time birth coordinates for the new particles according to a Poisson point process in $D$ with intensity measure $\langle\langle\mathcal{M}\rangle\rangle$ (interior birth sites) superposed with a Poisson point process on the boundary (boundary birth sites) with the intensity measure $\langle\langle\mathcal{M} ; \partial D\rangle\rangle$. Each interior birth site $x \in D$ emits two particles, moving with initial velocities $v^{\prime}$ and $v^{\prime \prime}$ chosen according to the joint distribution

$$
\theta_{x}^{\mathcal{M}}\left(d v^{\prime}, d v^{\prime \prime}\right):=\frac{\mathcal{M} \times \mathcal{M}\left(\left\{\left(l_{1}, l_{2}\right), l_{1} \cap l_{2} \in d x, v\left[l_{1}\right] \in d v^{\prime}, v\left[l_{2}\right] \in d v^{\prime \prime}\right\}\right)}{2\langle\langle\mathcal{M}\rangle\rangle(d x)}
$$

where $v[l]$ is the velocity of a particle whose space-time trajectory is represented by the straight line $l$. Note that this is equivalent to choosing the directions of the straight lines representing the space-time trajectories of the emitted particles according to the distribution of the typical angle between two lines of $\Lambda^{\mathcal{M}}$ at $x$, that is to say the angle arising by 
conditioning $\Lambda^{\mathcal{M}}$ on containing two lines intersecting at $d x$, see also Sections 3 and 4 in [2] and the references therein. In other words, (7) implies that the interior birth at $l_{1} \cap l_{2}$ in $D$ of two particles moving along straight lines $l_{1}$ and $l_{2}$ respectively happens with probability $\mathcal{M}\left(d l_{1}\right) \mathcal{M}\left(d l_{2}\right)$. Each boundary birth site $x \in \partial D$ yields one particle with initial speed $v$ determined according to the distribution $\theta_{x}^{\mathcal{M} ; \partial}(d v)$ identified by requiring that the direction of the line entering $D$ at $x$ and representing the space-time trajectory of the emitted particle be chosen according to the distribution of a straight line $l \in \Lambda^{\mathcal{M}}$ conditioned on the event $\{x=\operatorname{in}(l, D)\}$, that is to say

$$
\theta_{x}^{\mathcal{M} ; \partial}(d v):=\frac{\mathcal{M}(\{l, \operatorname{in}(l, D) \in d x, v[l] \in d v\})}{\langle\langle\mathcal{M} ; \partial D\rangle\rangle(d x)} .
$$

Again, this means that the boundary birth at $l \cap \partial D$ of a particle moving along a straight line $l$ occurs with probability $\mathcal{M}(d l)$.

All the particles evolve independently in time according to the following rules.

(E1) Between the critical moments listed below each particle moves freely with constant velocity so that $d y=v d t$,

(E2) When a particle touches the boundary $\partial D$, it dies,

(E3) In case of a collision of two particles (equal spatial coordinates $y$ at some moment $t$ with $(t, y) \in D)$, both of them die,

(E4) The time evolution of the velocity $v_{t}$ of an individual particle is given by the following pure-jump inhomogeneous Markov process: denoting by $l_{t}$ the straight line extending the present segment of the space-time trajectory of the particle at time $t$ so that $v_{t}=v\left[l_{t}\right]$, we have

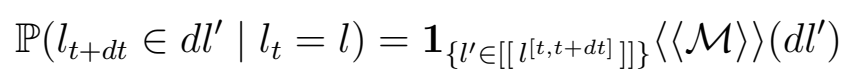

with $l^{[t, t+d t]}$ standing for the segment along $l$ between time moments $t$ and $t+d t$.

The evolution rule (E4) can be interpreted as follows: in the course of its movement along a short line segment $l^{[t, t+d t]}$ a particle turns and starts moving along another straight line $l^{\prime} \in\left[\left[l^{[t, t+d t]}\right]\right]$ with probability $\langle\langle\mathcal{M}\rangle\rangle\left(d l^{\prime}\right)$ which agrees with the interpretation of $\mathcal{M}$ as the line activity measure as discussed above. 
The following theorem shows that the polygonal field obtained in the course of the above dynamic construction is in fact $\mathcal{A}_{D}^{\mathcal{M}}=\mathcal{A}_{D}^{\mathcal{M} ; 1}$, in analogy with the results of Arak \& Surgailis [2], Section 4, for homogeneous fields.

Theorem 1 The polygonal field traced by the particle system constructed above coincides in distribution with $\mathcal{A}_{D}^{\mathcal{M}}=\mathcal{A}_{D}^{\mathcal{M} ; 1}$. Moreover, we have

$$
\mathcal{Z}_{D}^{\mathcal{M} ; 1}=\exp (\langle\langle\mathcal{M}\rangle\rangle(D))
$$

Proof We pick some $\gamma \in \Gamma_{D}$ and calculate the probability that the outcome of the above dynamic construction falls into $d \gamma$. To this end, we note that:

- Each edge $e \in \operatorname{Edges}(\gamma)$ with initial vertex (lower time coordinate) lying on $\partial D$ contributes to the considered probability the factor $\mathcal{M}(d l[e])$ (boundary birth of a particle tracing the edge) times $\exp (-\mathcal{M}([[e]]))$ (no velocity updates along $e$ ).

- Each of the two edges $e_{1}, e_{2} \in \operatorname{Edges}(\gamma)$ steming from a common interior birth site $l\left[e_{1}\right] \cap l\left[e_{2}\right]$ yields the factor $\mathcal{M}\left(d l\left[e_{i}\right]\right), i=1,2$, (coming from the birth probability) times $\exp \left(-\mathcal{M}\left(\left[\left[e_{i}\right]\right]\right)\right)$ (no velocity updates along $\left.e_{i}\right)$,

- Each of the edges $e \in \operatorname{Edges}(\gamma)$ arising due to a velocity update of a particle yields the factor $\mathcal{M}(d l[e])$ (velocity update probability) times $\exp (-\mathcal{M}(d l[e])$ ) (no velocity updates along $e$ ),

- The absence of interior birth sites in $D \backslash \gamma$ yields the factor $\exp (-\langle\langle\mathcal{M}\rangle\rangle(D))$,

- Finally, the absence of boundary birth sites at $\partial D \backslash \gamma$ yields the additional factor $\exp (-\langle\langle\mathcal{M} ; \partial D\rangle\rangle(D))=\exp (-\mathcal{M}(D))$.

Putting these observations together we conclude that the probability element of $\gamma$ being traced by the particle system is

$$
\frac{\exp \left(-L^{\mathcal{M}}(\gamma)\right)}{\exp (\langle\langle\mathcal{M}\rangle\rangle(D))} \frac{\prod_{e \in \operatorname{Edges}(\gamma)} \mathcal{M}(d l[e])}{\exp (\mathcal{M}(D))}=\frac{\exp \left(-L^{\mathcal{M}}(\gamma)\right)}{\exp (\langle\langle\mathcal{M}\rangle\rangle(D))} d \mathbb{P}\left(\gamma \in \Gamma_{D}\left(\Lambda^{\mathcal{M}}\right)\right),
$$

where the event $\left\{\gamma \in \Gamma_{D}(\Lambda)^{\mathcal{M}}\right\}$ is easily seen to correspond to the situation where the collection of edge-extending lines $\{l[e], e \in \operatorname{Edges}(\gamma)\}$ coincides with the collection of lines determined by $\Lambda_{D}^{\mathcal{M}}$. This shows that the field traced by the particles coincides in law with $\mathcal{A}_{D}^{\mathcal{M}}$ and that (9) holds, thus completing the proof of the theorem. 


\subsection{Conclusions from the dynamic representation}

The crucial property of polygonal fields $\mathcal{A}_{(\cdot)}^{\mathcal{M}}$ for $\beta=1$ is their consistency stated in Theorem 2 below and established using the dynamic representation from Theorem 1 in analogy with Arak \& Surgailis [2]. This is where the name consistent regime comes from. Another important feature of $\mathcal{A}_{(\cdot)}^{\mathcal{M}}$ is the explicit knowledge of its linear sections, see Theorem 2, which can be further used to obtain information about the first and second order correlation structure of the field, see Theorem 4 and Corollaries 1, 2 and 4.

Theorem 2 The polygonal field $\mathcal{A}_{D}^{\mathcal{M}}$ enjoys the following properties

Consistency: For bounded open convex $D^{\prime} \subseteq D \subseteq \mathbb{R}^{2}$ the field $\mathcal{A}_{D}^{\mathcal{M}} \cap D^{\prime}$ coincides in law with $\mathcal{A}_{D^{\prime}}^{\mathcal{M}}$. This allows us to construct the whole plane extension of the process $\mathcal{A}^{\mathcal{M}}$ such that for each bounded open convex $D \subseteq \mathbb{R}^{2}$ the field $\mathcal{A}_{D}^{\mathcal{M}}$ coincides in law with $\mathcal{A}^{\mathcal{M}} \cap D$.

Linear sections: For a straight line $l$ in $\mathbb{R}^{2}$ the collection of intersection points and intersection directions of $l$ with the edges of the polygonal field $\mathcal{A}^{\mathcal{M}}$ coincide in law with the corresponding collection for the Poisson line process $\Lambda^{\mathcal{M}}$.

Proof To establish the Consistency property, choose a bounded open convex set $D \subseteq$ $\mathbb{R}^{2}$ and a straight line $l$ intersecting $D$ and define $D^{\prime}$ to be the set of points of $D$ lying to the left from $l$ (lower time coordinates). Clearly then, from the dynamic representation Theorem 1 we conclude the Consistency statement for the so chosen $D$ and $D^{\prime}$. Noting that the dynamic representation is equally available upon rotating the space-time coordinate system we see that the Consistency holds as well upon cutting off the part of the set $D$ lying to the left of $l$. This means however that the consistency holds upon cutting off pieces of the original set with arbitrary straight lines - a repetitive use of this procedure and a possible passage to the limit allows us to carve from $D$ its arbitrary convex subset. This proves the Consistency claim. To establish the Linear sections statement pick a straight line $l$ and choose the space-time coordinate system so that $l$ coincides with its spatial axis. The Linear sections claim follows now from the form of the boundary birth mechanism in the dynamic representation in view of the Consistency property. 


\section{Generalised dynamic representation for consistent fields}

The dynamic construction of consistent polygonal fields, originating in the homogeneous set-up from Arak \& Surgailis [2] and discussed in Subsection 3 above, can be regarded as revealing increasing portions of the polygonal field in the course of the time flow. Under this interpretation, the portion of a polygonal field in a bounded open convex domain $D$ uncovered by time $t$ is precisely its intersection with $D_{t}=\bar{D} \cap(-\infty, t] \times \mathbb{R}_{+}$. The idea underlying our generalised version of dynamic representation developed in the present section is to replace the above family $D_{t}$ by some other time-increasing family of subsets of $D$, also denoted $D_{t}$ in the sequel, eventually covering the whole $D$, and to try to provide a natural construction of the polygonal field being gradually uncovered on the growing domain $D_{t}$ in the course of the time flow. We shall always assume that $D_{t}$ be convex for otherwise we would have to deal with situations where two or more disconnected parts of an edge of the field have been revealed which leads to unwanted and cumbersome dependencies along the segments connecting these parts. Taking this into account and having formal convenience in mind we impose the following natural assumptions on $D$ and $D_{t}, t \in[0,1]$,

(D1) $\left(D_{t}\right)_{t \in[0,1]}$ is a strictly increasing family of compact convex subsets of $\bar{D}=D \cup \partial D$.

(D2) $D_{0}$ is a single point $x$ in $\bar{D}=D \cup \partial D$.

(D3) $D_{1}$ coincides with $\bar{D}$.

(D4) The family $\left(D_{t}\right)_{t \in[0,1]}$ enjoys the property that $\mathcal{M}\left(\left\{l, \exists_{t \in[0,1]} \operatorname{card}\left(l \cap \partial D_{t}\right)>2\right\}\right)=0$, in particular $\bar{D}$ itself satisfies $\mathcal{M}(\{l, \operatorname{card}(l \cap \partial D)>2\})=0$.

(D5) $D_{t}$ is continuous in the usual Hausdorff metric on compacts.

Under these conditions, for $\mathcal{M}$-almost each $l \in[[D]]$ the intersection $l \cap D_{\tau_{l}}$ consists of precisely one point $\mathbb{A}(l)$, where $\tau_{l}=\inf \left\{t \in[0,1], D_{t} \cap l \neq \emptyset\right\}$. The point $\mathbb{A}(l)$ will be referred to as the anchor point for $l$, this induces the anchor mapping $\mathbb{A}:[[D]] \rightarrow D$. Consider now the following dynamics in time $t \in[0,1]$, with all updates given by the rules below performed independently of each other.

(GE:Initialise) Begin with empty field at time 0, 
(GE1) Between critical moments listed below, during the time interval $[t, t+d t]$ the unfolding field edges in $D_{t}$ reaching $\partial D_{t}$ extend straight to $D_{t+d t} \backslash D_{t}$,

(GE2) When a field edge hits the boundary $\partial D$, it stops growing in this direction (recall that $\mathcal{M}$-almost everywhere the intersection of a line with $\partial D$ consists of at most two points),

(GE3) When two unfolding field edges intersect in $D_{t+d t} \backslash D_{t}$, they are not extended any further beyond the intersection point (stop growing in the direction marked by the interesction point),

(GE4) A field edge extending along $l \in\left[\left[D_{t}\right]\right]$ updates its direction during $[t, t+d t]$ and starts unfolding along $l^{\prime} \in\left[\left[l^{[t, t+d t]}\right]\right]$, extending away from the anchor point $\mathbb{A}\left(l^{\prime}\right)$, with probability $\mathcal{M}\left(d l^{\prime}\right)$, where $l^{[t, t+d t]}:=l \cap\left(D_{t+d t} \backslash D_{t}\right)$. Directional updates of this type are all performed independently,

(GE:LineBirth) Whenever the anchor point $\mathbb{A}(l)$ of a line $l$ falls into $D_{t+d t} \backslash D_{t}$, the line $l$ is born at the time $t$ at its anchor point with probability $\mathcal{M}(d l)$, whereupon it begins extending in both directions with the growth of $D_{t}$ (recall that $l$ is $\mathcal{M}$-almost always tangential to $\partial D_{t}$ here),

(GE:VertexBirth) For each intersection point of lines $l_{1}$ and $l_{2}$ falling into $D_{t+d t} \backslash D_{t}$, the pair of field lines $l_{1}$ and $l_{2}$ is born at $l_{1} \cap l_{2}$ with probability $\mathcal{M}\left(d l_{1}\right) \mathcal{M}\left(d l_{2}\right)$, whereupon both lines begin unfolding in the directions away from their respective anchor points $\mathbb{A}\left(l_{1}\right)$ and $\mathbb{A}\left(l_{2}\right)$.

Observe that the evolution rule (GE:VertexBirth) means simply that pairs of lines are born at birth sites distributed according to a Poisson point process in $D$ with intensity measure $\langle\langle\mathcal{M}\rangle\rangle$, in analogy to the standard dynamic representation in Subsection 3.1. The essential difference though is that here the pairs of lines emitted from a birth site extend away from their respective anchor points rather than always in the direction determined by a single time axis. Further, the line birth events given by (GE:LineBirth) replace the boundary birth events in the standard dynamic representation. It is also worth noting that if we choose the family $D_{t}$ so that $D_{t}:=\bar{D} \cap\left(-\infty,(1-t) x_{\min }+t x_{\max }\right] \times \mathbb{R}_{+}$, where $x_{\min }$ and $x_{\max }$ are the minimal and maximal x-coordinates of a point in $D$ (assume that $\bar{D}$ contains exactly one point with $\mathrm{x}$-coordinate $x_{\min }$ and that $\mathcal{M}$ assigns zero mass to 
the set of vertical lines), the generalised dynamic representation (GE) coincides with the standard Arak \& Surgailis one determined by rules (E1-4) in Subsection 3.1 above, and we have $\mathbb{A}(l)=\operatorname{in}(l, D)$.

In analogy with the corresponding result for the usual dynamic construction, as established in the proof of Theorem 1, we show that the field resulting from the above (GE) construction does coincide in law with $\mathcal{A}_{D}^{\mathcal{M}}$.

Theorem 3 The random contour ensemble resulting from the above construction (GE) coincides in law with $\mathcal{A}_{D}^{\mathcal{M}}$.

Proof The proof is very similar to that of Theorem 1. We pick some $\gamma \in \Gamma_{D}$ and calculate the probability that the outcome of the above dynamic construction falls into $d \gamma$. To this end, we note that:

- Each edge $e \in \operatorname{Edges}(\gamma)$ containing the anchor point $\mathbb{A}(l[e])$ and hence resulting from a line birth event due to the rule (GE:LineBirth), contributes to the considered probability the factor $\mathcal{M}(d l[e])$ (line birth probability for $l[e]$ ) times $\exp (-\mathcal{M}([[e]])$ ) (no directional updates along $e$ ),

- Each of the two edges $e_{1}, e_{2} \in \operatorname{Edges}(\gamma)$ steming from a common interior birth vertex $l\left[e_{1}\right] \cap l\left[e_{2}\right]$ yields the factor $\mathcal{M}\left(d l\left[e_{i}\right]\right), i=1,2$, (coming from the vertex birth probability due to the rule (GE:VertexBirth)) times $\exp \left(-\mathcal{M}\left(\left[\left[e_{i}\right]\right]\right)\right)$ (no directional updates along $e_{i}$ ),

- Each of the edges $e \in \operatorname{Edges}(\gamma)$ arising due to a directional update in (GE4) yields the factor $\mathcal{M}(d l[e])$ (directional update probability) times $\exp (-\mathcal{M}(d l[e]))$ (no directional updates along $e$ ),

- The absence of interior birth sites in $D \backslash \gamma$ yields the factor of $\exp (-\langle\langle\mathcal{M}\rangle\rangle(D))$,

- Finally, the absence of line birth events for all lines in $[[D]]$ except for the finite collection $\{l[e], e \in \operatorname{Edges}(\gamma)\}$ yields the additional factor $\exp (-\mathcal{M}(D))$.

Putting these observations together we conclude that the probability element of $\gamma$ resulting from the generalised construction above is

$$
\frac{\exp \left(-L^{\mathcal{M}}(\gamma)\right)}{\exp (\langle\langle\mathcal{M}\rangle\rangle(D))} \frac{\prod_{e \in \operatorname{Edges}(\gamma)} \mathcal{M}(d l[e])}{\exp (\mathcal{M}(D))}=\frac{\exp \left(-L^{\mathcal{M}}(\gamma)\right)}{\exp (\langle\langle\mathcal{M}\rangle\rangle(D))} d \mathbb{P}\left(\gamma \in \Gamma_{D}\left(\Lambda^{\mathcal{M}}\right)\right)
$$


and thus the field obtained by this construction coincides in law with $\mathcal{A}_{D}^{\mathcal{M}}$ as required. This completes the proof of the theorem.

\section{Correlations in the consistent regime}

In the present section we use the dynamic construction and its generalised version to describe the correlation structure of the consistent field $\mathcal{A}^{\mathcal{M}}$. Due to the polygonal nature of the considered field the natural object of our interest are the edge correlations

$$
\sigma^{\mathcal{M}}\left[d l_{1}, x_{1} ; \ldots ; d l_{k}, x_{k}\right]:=\mathbb{P}\left(\forall_{i=1}^{k} \exists_{e \in \operatorname{Edges}\left(\mathcal{A}^{\mathcal{M}}\right)} \pi_{l_{i}}\left(x_{i}\right) \in e, l[e] \in d l_{i}\right),
$$

where $l_{1}, \ldots, l_{k}$ are straight lines and $\pi_{l_{i}}$ is the orthogonal projection on $l_{i}$. In almost all cases below we shall be interested in correlations with $x_{i} \in l_{i}$, in which case $\sigma^{\mathcal{M}}\left[d l_{1}, x_{1} ; \ldots\right.$; $\left.d l_{k}, x_{k}\right]$ can be interpreted as the probability element that the polygonal field $\mathcal{A}^{\mathcal{M}}$ passes through points $x_{i}$ in the directions determined by the respective lines $l_{i}, i=1, \ldots, k$. For general $x_{i}$, not necessarily lying on $l_{i}$, the $k$-fold correlation $\sigma^{\mathcal{M}}\left[d l_{1}, x_{1} ; \ldots ; d l_{k}, x_{k}\right]$ is the probability that the polygonal field passes through points $\pi_{l_{i}}\left(x_{i}\right)$ in the directions determined by the respective lines $l_{i}, i=1, \ldots, k$.

\subsection{First and second order edge correlations}

The first and second order edge correlations are easily determined using the Linear sections statement of Theorem 2 concluded from the standard dynamic representation.

Theorem 4 We have for $x \in l$

$$
\sigma^{\mathcal{M}}[d l, x]=\mathcal{M}(d l)
$$

and, for $x_{1} \in l_{1}, x_{2} \in l_{2}$,

$$
\sigma^{\mathcal{M}}\left[d l_{1}, x_{1} ; d l_{2}, x_{2}\right]= \begin{cases}\mathcal{M}\left(d l_{1}\right) \mathcal{M}\left(d l_{2}\right), & \text { if } l_{1} \neq l_{2}, \\ \exp \left(-2 \mathcal{M}\left(\left[\left[\overline{x_{1} x_{2}}\right]\right]\right)\right) \mathcal{M}(d l), & \text { if } l_{1}=l_{2}\end{cases}
$$

where $\overline{x_{1} x_{2}}$ is the segment joining $x_{1}$ to $x_{2}$.

Proof The statement for $\sigma^{\mathcal{M}}[d l, x]$ and $\sigma^{\mathcal{M}}\left[d l_{1}, x_{1} ; d l_{2}, x_{2}\right], l_{1} \neq l_{2}$, is a direct consequence of the Linear sections part of Theorem 2 and of the properties of the Poisson line process $\Lambda^{\mathcal{M}}$. To find $\sigma^{\mathcal{M}}\left[d l, x_{1} ; d l, x_{2}\right]$ for $x_{1}, x_{2} \in l$ note that in order to have an edge $e$ of the field passing through both $x_{1}$ and $x_{2}$ along $l$, we have to ensure that 
- There is $e \in \operatorname{Edges}\left(\mathcal{A}^{\mathcal{M}}\right)$ such that $l[e] \in d l$ and $x_{1}$, which happens with probability $\mathcal{M}(d l)$ in view of Linear sections in Theorem 2,

- There are no other edges of the field crossing $e$ between $x_{1}$ and $x_{2}$, which happens with probability $\exp \left(-2 \mathcal{M}\left(\left[\left[\overline{x_{1} x_{2}}\right]\right]\right)\right)$. Indeed, to see it choose the spatial axis in the standard dynamic representation in Subsection 3.1 very close to $l$ (not exactly $l$ to keep the velocity of the particle tracing $e$ finite though very large) and observe that $e$ can be crossed by

- space-time trajectories of particles coming from the past, which happens with probability $1-\exp \left(-\mathcal{M}\left(\left[\left[\overline{\bar{x}_{1} x_{2}}\right]\right]\right)\right)[1+o(1)]$ by the Linear sections property,

- edges arising due to velocity updates along $\overline{x_{1} x_{2}}$, which happens with probability $\left.1-\exp \left(-\mathcal{M}\left(\left[\overline{x_{1} x_{2}}\right]\right]\right)\right)[1+o(1)]$ by the dynamic rule $(\mathbf{E} 4)$.

Since the velocity update events in (E4) are independent of the past particle configuration, letting the spatial axis approach $l$ we obtain the required conclusion.

Combining the factors listed above we obtain the required formula for $\sigma^{\mathcal{M}}\left[d l, x_{1} ; d l, x_{2}\right]$, thus completing the proof of the Theorem.

\subsection{Higher order edge correlations for general activity measures}

To describe the higher order correlation structure of the field $\mathcal{A}^{\mathcal{M}}$ we need the full power of the more flexible generalised dynamic representation. Consider a collection $\left(l_{1}, x_{1}\right),\left(l_{2}, x_{2}\right)$, $\ldots,\left(l_{k}, x_{k}\right), k \geq 1$, of pairwise different lines $l_{i}$ and points $x_{i}$ with $x_{i} \in l_{i}$ and $x_{i} \notin l_{j}$ for $j \neq i$. Such collections are said to be in general position below and such assumption will be imposed an all collections $\left(l_{i}, x_{i}\right)$ considered in this subsection, often without a further mention. Also, throughout this subsection we always assume for formal convenience that all $x_{i}, i=1, \ldots, n$, are contained in a bounded open convex set $D$, playing the usual role of the field domain. Clearly, the particular choice of $D$ is irrelevant due to the consistency of the field $\mathcal{A}^{\mathcal{M}}$.

We say that the edge correlations of the field $\mathcal{A}^{\mathcal{M}}$ factorise on a collection $\left(l_{i}, x_{i}\right)_{i=1}^{k}$ if $\sigma^{\mathcal{M}}\left[d l_{1}, x_{1} ; \ldots ; d l_{k}, x_{k}\right]$ coincides with the product $\prod_{i=1}^{k} \sigma^{\mathcal{M}}\left[d l_{i}, x_{i}\right]$. Moreover, we say that the collective factorisation of correlations holds for a family $\left(l_{i}, x_{i}\right)_{i=1}^{k}$ if the correlations 
factorise for $\left(l_{i}, x_{i}\right)_{i=1}^{k}$ and all its sub-collections. In view of Theorem $4\left(l_{i}, x_{i}\right)_{i=1}^{k}$ collectively factorises iff

$$
\sigma^{\mathcal{M}}\left[d l_{i_{1}}, x_{i_{1}} ; \ldots ; d l_{i_{m}}, x_{i_{m}}\right]=\prod_{j=1}^{m} \mathcal{M}\left(d l_{i_{j}}\right)
$$

for all sub-collections $\left(l_{i_{j}}, x_{i_{j}}\right)_{i=1}^{m}$.

General collective factorisation problem and precedence graphs Our main objective in the present subsection is to find general conditions characterising collections $(\bar{l}, \bar{x})$ in general position enjoying the collective factorisation property. To see what may be plausible answers to this question let us make the following basic observations very helpful in interpreting the geometry of the higher order edge correlations. For a collection $(\bar{l}, \bar{x})=\left(l_{1}, x_{1} ; \ldots ; l_{k}, x_{k}\right), x_{i} \in l_{i}$, in general position by $\Gamma(\bar{l}, \bar{x})$ we shall mean the family of admissible polygonal configurations $\gamma$ in the plane consisting of $k$ edges $e_{1}, \ldots, e_{k}$ such that $e_{i}$ lies on $l_{i}$ and contains $x_{i}$ for all $i=1, \ldots, k$. Looking at the structure of the family $\Gamma(\bar{l}, \bar{x})$ is closely related to the following natural problem which marks its presence in various domains ranging from kid games to studies on human and computer vision, see [5] and the references therein: given the collection $(\bar{l}, \bar{x})=\left(l_{i}, x_{i}\right)_{i=1}^{k}$ draw a family of closed curves (here a polygonal configuration) such that each point $x_{i}$ lies on one of the curves and the direction of the curve at $x_{i}$ is determined by $l_{i}$. Clearly, the solution to this problem is non-unique in general, yet if we require in addition that the configuration we draw belong to $\Gamma(\bar{l}, \bar{x})$, that is to say we may only draw over the lines $l_{i}$ and on each of these lines we have to draw precisely one segment of non-zero length, then it is often the case that $\Gamma(\bar{l}, \bar{x})$ is a singleton. Now, assume that all points $x_{i}$ as well as the intersection points $y_{i, j}$ for $l_{i}$ and $l_{j}$ lie very close to each other, say they are all contained in a disk of very small radius $r$, then it follows from (2) and (3) combined with the consistency property of the field that

$$
\sigma^{\mathcal{M}}\left[d l_{1}, x_{1} ; \ldots ; d l_{k}, x_{k}\right]=N(\bar{l}, \bar{x}) \mathcal{M}\left(d l_{1}\right) \ldots \mathcal{M}\left(d l_{k}\right)\left(1+o_{r}(1)\right)
$$

where $N(\bar{l}, \bar{x})$ is the cardinality of $\Gamma(\bar{l}, \bar{x})$. Indeed, to see it note that the Boltzmann weight $\exp \left(-L^{\mathcal{M}}(\cdot)\right)$ tends to 1 as $r \rightarrow 0$. Thus, the factorisation holds in small $r$ asymptotics precisely when $\Gamma(\bar{l}, \bar{x})$ is a singleton. As will be discussed in the sequel, there are many collections for which $N(\bar{l}, \bar{x})=0$ or $N(\bar{l}, \bar{x})>1$. In particular, it is not the case that the factorisation holds for all collections in general position. Neither can it be hoped 
though that the formula (12) holds in general non-asymptotic regime: as we shall show in Theorem 11 in Section 7 in the particular case of rectangular fields, when the distances between $x_{i}$ 's get large, the correlations converge exponentially fast to the product, that is to say the polygonal field exhibits exponential decay of dependencies. To conclude these considerations, we say that $\left(l_{i}, x_{i}\right)_{i=1}^{k}$ enjoys collective factorisation on all scales iff $\left(\alpha l_{i}, \alpha x_{i}\right)_{i=1}^{k}$ factorises collectively for each $\alpha>0$, where by $(\alpha l, \alpha x)$ we understand the re-scaled version of $(l, x)$ with scaling factor $\alpha$. Then the above discussion shows that

Lemma 1 For a collection $(\bar{l}, \bar{x})=\left(l_{i}, x_{i}\right)_{i=1}^{k}$ in general position a necessary condition to enjoy collective factorisation on all scales is that $N(\bar{l}, \bar{x})=1$ and $N\left(\bar{l}^{\prime}, \bar{x}^{\prime}\right)=1$ for all non-empty sub-collections $\left(\bar{l}^{\prime}, \bar{x}^{\prime}\right)$ of $(\bar{l}, \bar{x})$.

To reformulate this condition in more tangible terms, we build for each family $\left(l_{i}, x_{i}\right)_{i=1}^{k}$ its precedence graph $\mathcal{G}\left[l_{1}, x_{1} ; \ldots ; l_{k}, x_{k}\right]$ as follows. We split each $l_{i}$ at $x_{i}$ into two half-lines both oriented in the directions away from $x_{i}$. This creates a directed graph $\mathcal{G}\left[l_{1}, x_{1} ; \ldots ; l_{k}, x_{k}\right]$ whose vertices are the generating points $x_{i}$ and the intersection points $y_{i, j}$ of respective pairs of lines $l_{i}, l_{j}$. As we shall see below, the name precedence graph comes from its relationship to the order in which the points of $\bigcup_{i=1}^{k} l_{i}$ are revealed in the course of a suitable instance of the generalised graphical construction. For now, we claim that

Lemma 2 For a collection $(\bar{l}, \bar{x})=\left(l_{i}, x_{i}\right)_{i=1}^{k}$ in general position the following conditions are equivalent

$$
\text { 1. } N(\bar{l}, \bar{x})=1 \text { and } N\left(\bar{l}^{\prime}, \bar{x}^{\prime}\right)=1 \text { for all non-empty sub-collections of }(\bar{l}, \bar{x}) \text {, }
$$

2. The precedence graph $\mathcal{G}\left[l_{1}, x_{1} ; \ldots ; l_{k}, x_{k}\right]$ is acyclic.

Proof To show that the first condition implies the second one note that if the precedence graph contains a cycle of length $m$ built by $\left(l_{i_{1}}, x_{i_{1}} ; \ldots ; l_{i_{m}}, x_{i_{m}}\right)$, then for the sub-collection $\left(\bar{l}^{\prime}, \bar{x}^{\prime}\right)=\left(l_{i_{j}}, x_{i_{j}}\right)_{j=1}^{m}$ we have $N\left(\bar{l}^{\prime}, \bar{x}^{\prime}\right)=0$ if $m$ is odd and $N\left(\bar{l}^{\prime}, \bar{x}^{\prime}\right)=2$ if $m$ is even.

To prove the inverse implication assume that the precedence graph $\mathcal{G}\left[l_{1}, x_{1} ; \ldots ; l_{k}, x_{k}\right]$ contains no cycles and observe that this induces a partial ordering on $\bigcup_{i=1}^{k} l_{i}$ in which the $x_{i}$ 's are minimal points, each $x_{i}$ first on its corresponding line $l_{i}$ and with the remaining points on $l_{i}$ ordered according to the natural linear orderings directed away from $x_{i}$ on the two half-lines. This will be referred to as the structural ordering for the collection $\left(l_{i}, x_{i}\right)_{i=1}^{k}$ in the sequel. Consider the following incremental construction of a graph belonging to 
$\Gamma(\bar{l}, \bar{x})$, where at each step we obtain a collection of $k$ segments $\iota_{i} \subseteq l_{i}, i=1, \ldots, k$ with non-intersecting interiors but possibly sharing endpoints and possibly degenerated to $x_{i}$ 's, eventually yielding the entire graph under construction.

1. begin with $\iota_{i}:=\left\{x_{i}\right\}, i=1, \ldots, k$,

2. choose an intersection point $y_{i, j}$ which:

(a) belongs to neither $\iota_{i}$ nor $\iota_{j}$ and hence to neither of the remaining segments $\iota_{l}$.

(b) enjoys the property that extending both $\iota_{i}$ and $\iota_{j}$ to contain $y_{i, j}$ creates no Tshaped or X-shaped nodes (vertices of order three or four respectively), which is equivalent to both $\iota_{i}$ and $\iota_{j}$ having their endpoints pointing towards $y_{i, j}$ not shared with any other segment $\iota_{l}$. (Such endpoints will be referred to as loose ends below. Note that by definition of our construction procedure the only possible loose end on $\iota_{i}$ is $x_{i}$.)

(c) is minimal with the above two properties.

with possible ties broken in an arbitrary way,

3. extend both segments $\iota_{i}$ and $\iota_{j}$ to contain $y_{i, j}$ - this action will be referred to as adding $y_{i, j}$ to the graph for short in the sequel of this argument,

4. return to 2. unless no more $y_{i, j}$ 's can be added,

5. whenever a segment $\iota_{l}$ of the constructed graph ends with a loose node, that is to say a vertex of order one, extend this segment to the half-line in the direction of the node.

In other words, we initialise our graph under construction with the set of generating points $\left\{x_{1}, \ldots, x_{k}\right\}$ whereupon we let it grow along the lines $l_{i}$, adding subsequent intersection points $y_{i, j}$ in the order determined by the structural ordering whenever this does not violate the usual constraints imposed on a polygonal configuration, and discarding those $y_{i, j}$ 's whose addition would violate these constraints. It is easily seen that this procedure yields in a finite number of steps a graph belonging to $\Gamma(\bar{l}, \bar{x})$, thus in particular $\Gamma(\bar{l}, \bar{x}) \neq \emptyset$ and $N(\bar{l}, \bar{x}) \geq 1$. To show that $N(\bar{l}, \bar{x}) \leq 1$ note that in fact all graphs in $\Gamma(\bar{l}, \bar{x})$ can be obtained by the above procedure. Indeed, this is seen inductively. First, all $x_{i}$ 's have to 
belong to such a graph. Second, whenever a graph in $\Gamma(\bar{l}, \bar{x})$ contains some configuration of $\iota_{i}$ 's as its (sub)segments and some of these $\iota_{i}$ 's have loose ends $x_{i}$ 's, each of such $\iota_{i}$ 's has to be extended, either up to intersection with the extension of another segment or to the entire half-line if such an intersection is not available. This is because no loose ends can be present in the final graph and because we cannot hope that the potential loose end $x_{i}$ on $\iota_{i}$ could be possibly reached by another edge $\iota_{j}, j \neq i$, since $x_{i} \notin l_{j}, j \neq i$. This means that at least one intersection point $y_{i, j}$ as in 2.(a,b,c) has to be added to the graph as in 2 . Consequently, two different graphs in $\Gamma(\bar{l}, \bar{x})$ could only arise in the course of two distinct instances of the above procedure, with different choices of minimal points $y_{i, j}$ in step 2 . We argue that the so constructed graphs necessarily coincide though. To see it let us make the following observations

- If at a certain stage of the construction some intersection point $y_{i, j}$ becomes admissible for 2.(a,b,c), there is no way to make it inadmissible whatever choices be made in the sequel of the construction save for adding $y_{i, j}$ to the graph. Indeed, to make $y_{i, j}$ inadmissible in the sequel of the construction we would have to close either $\iota_{i}$ or $\iota_{j}$ by intersecting its extension with an extension of some other $\iota_{l}$ before $\iota_{i}$ or $\iota_{j}$ hits $y_{i, j}$, which is not possible by the minimality of $y_{i, j}$ in 2.(a,b,c).

- If at a certain stage of the construction some intersection point $y_{i, j}$ becomes admissible for 2.(a,b,c), there is no way to make it inadmissible by adding some other intersection points in the prequel of the construction in agreement with the rule 2 . (without removing those already present though). Indeed, to make $y_{i, j}$ inadmissible in this way we would have to have added some other intersection point $y$ on $\iota_{i}$ or $\iota_{j}$ prior to the considered construction stage, but if this were possible this point $y$ would have to reach its admissibility before $y_{i, j}$ and in view the previous observation there would be no way to make it inadmissible before $y_{i, j}$ reaches its admissibility, which would contradict the minimality of $y_{i, j}$ in $2 .(\mathrm{a}, \mathrm{b}, \mathrm{c})$ at the moment of becoming admissible.

Consequently, regardless of any particular sequence of choices in a given instance of our construction, the points admissible for 2.(a,b,c) in the very first step of the construction (call them the first generation) eventually have to be added in view of the first observation above. Next, by the first and second observation above, all points which become admissible upon adding just the first generation (call them the second generation) also have to be added at some stage of the construction regardless of the particular sequence of choices 
made. Proceeding further this way we conclude inductively that the so-defined generations of all orders will eventually be added to the graph. However such generation-wise addition of intersection points (plus possible extensions to half-lines at the end of the procedure) is also an instance of our incremental construction and it yields a valid graph belonging to $\Gamma(\bar{l}, \bar{x})$ which, in view of the above discussion, enjoys the property of being contained in any other graph obtained in any instance of our construction. It remains to observe that a valid graph in $\Gamma(\bar{l}, \bar{x})$ cannot be further extended by our construction. All this means that the results of all possible instances of our construction coincide and the order in which the points $y_{i, j}$ are considered in 2 . is irrelevant. This way, we have shown that $N(\bar{l}, \bar{x})=1$ as required.

We ask whether the necessary precedence graph acyclicity condition stated in combined Lemmas 1 and 2 is also a sufficient condition for collective factorisation of correlations (on all scales). Below we are going to show that the answer to this question is positive for rectangular fields, see Subsection 5.3 and Theorem 6 there. For general fields we were only able to establish collective edge factorisation under a somewhat stronger sufficient condition though, see Theorem 5, and we do not know at present if this condition can be weakened.

General sufficient condition for collective factorisation We proceed with the general case first. To this end, we note that the generalised dynamic construction with its (GE:LineBirth) rule allows us to conclude:

Lemma 3 Assume that $\left(l_{i}, x_{i}\right)_{i=1}^{k}$ is a collection of pairwise distinct lines and points in general position and such that $l_{1}$ does not hit the convex hull $\operatorname{conv}\left(\left\{x_{2}, \ldots, x_{k}\right\}\right)$. Then

$$
\sigma^{\mathcal{M}}\left[d l_{1}, x_{1} ; \ldots ; d l_{k}, x_{k}\right]=\mathcal{M}\left(d l_{1}\right) \sigma^{\mathcal{M}}\left[d l_{2}, x_{2} ; \ldots ; d l_{k}, x_{k}\right]
$$

Indeed, under the assumptions of the corollary, by standard geometry it is always possible to construct an increasing family $\left(D_{t}\right), t \in[0,1]$, of convex compacts satisfying rules (D15) as in Section 4 and such that $D_{t}$ covers the whole of $\operatorname{conv}\left(\left\{x_{2}, \ldots, x_{k}\right\}\right)$ before hitting $l_{1}$ and that $\mathbb{A}\left(l_{1}\right)=x_{1}$, that is to say $x_{1}$ is the first point of $l_{1}$ hit by $D_{t}$. This yields the required statement by the consistency of the field and by the (GE:LineBirth) rule. This way of thinking suggests a natural sufficient condition for collective factorisation to hold under general activity measures, namely that there exist an increasing family $\left(D_{t}\right), t \in[0,1]$, of convex compacts as in Section 4 and such that $x_{i}=\mathbb{A}\left(l_{i}\right)$ for all $1 \leq i \leq k$, that is to say $x_{i}$ 
is the point at which $l_{i}$ is first hit by $D_{t}$. However, the so formulated collective factorisation condition is rather untractable, therefore we look for its equivalent reformulation in more tangible terms. To this end we augment the precedence graph $\mathcal{G}\left[l_{1}, x_{1} ; \ldots ; l_{k}, x_{k}\right]$ as follows. For each two lines $l_{i}, l_{j}$ intersecting at some $y_{i, j}$ we note that $l_{i}$ and $l_{j}$ divide the plane into four regions, one with both $x_{i}$ and $x_{j}$ on its boundary, two with either $x_{i}$ or $x_{j}$, and finally one, termed the trap region, with neither. Now, if some $x_{m}$ falls into such a trap region, it is easily seen that for each family $D_{t}$ such that $\mathbb{A}\left(l_{i}\right)=x_{i}, i=1, \ldots, k$, we must have $y_{i j}$ hit by $D_{t}$ prior to $x_{m}$. Indeed, if at a certain time $D_{t}$ contains $x_{m}$, then if by that time it contains either of the points $x_{i}$ or $x_{j}$, it has to intersect both $l_{i}$ and $l_{j}$ and thus contain both $x_{i}, x_{j}$ and hence also $y_{i j}$ by convexity. On the other hand if $D_{t}$ hits $x_{m}$ before hitting any of $x_{i}$ and $x_{j}$ then we cannot have simultaneously $\mathbb{A}\left(l_{i}\right)=x_{i}$ and $\mathbb{A}\left(l_{j}\right)=x_{j}$. Taking this into account we add in $\mathcal{G}\left[l_{1}, x_{1} ; \ldots ; l_{k}, x_{k}\right]$ a directed trap edge from $y_{i j}$ to $x_{m}$ for each $i, j, m$ as above. Denote the resulting augmented directed graph by $\mathcal{G}^{+}\left[l_{1}, x_{1} ; \ldots ; l_{k}, x_{k}\right]$. It is important to observe that, as follows by its construction and the above discussion, the orientation of edges in this graph indicates the order in which its vertices are hit by the sought for increasing family $D_{t}$, should it exist. Consequently, the acyclicity of the augmented precedence graph is a necessary condition for the existence of such $D_{t}$. In the proof of the following crucial theorem we show that it is also a sufficient condition.

Theorem 5 Assume that $\left(l_{i}, x_{i}\right)_{i=1}^{k}$ is a collection of pairwise distinct lines and points in general position such that the augmented precedence graph $\mathcal{G}^{+}\left[l_{1}, x_{1} ; \ldots ; l_{k}, x_{k}\right]$ is acyclic. Then $\left(l_{i}, x_{i}\right)_{i=1}^{k}$ admits collective factorisation of correlations on all scales.

Proof In context of the discussion above and in view of the generalised graphical construction and its (GE:LineBirth) rule as used in Lemma 3, it suffices to show that there exists an increasing family $D_{t}$ such that $\mathbb{A}\left(l_{i}\right)=x_{i}$ for all $i=1, \ldots, k$. Now, to establish the existence of such a family it is enough to know that having $\mathcal{G}^{+}\left[l_{1}, x_{1} ; \ldots ; l_{k}, x_{k}\right]$ acyclic implies the existence of a permutation $\left(s_{i}\right)_{i=1}^{k}$ of indices such that $l_{s_{i+1}}$ does not hit the convex hull $\operatorname{conv}\left(\left\{x_{s_{1}}, \ldots, x_{s_{i}}\right\}\right), i=1, \ldots, k-1$. Indeed, the family $D_{t}$ is then easily constructed by induction in $k$ : it starts growing from $x_{s_{1}}$ whereupon it hits the consecutive points $x_{s_{2}}, x_{s_{3}}, \ldots$, and since $l_{s_{k+1}}$ is disjoint with $\operatorname{conv}\left(\left\{x_{s_{1}}, \ldots, x_{s_{k}}\right\}\right)$, the family $D_{t}$ can be chosen so that it does not hit $l_{s_{k+1}}$ until it reaches all previous lines $l_{s_{i}}, i \leq k$, and so that $\mathbb{A}\left(l_{s_{i}}\right)=x_{s_{i}}$ for $i \leq k$ (by induction hypothesis) and then $\mathbb{A}\left(l_{s_{k+1}}\right)=x_{s_{k+1}}$ (again by disjointness of $l_{s_{k+1}}$ with the convex hull of preceding $x_{s_{i}}$ 's). This can be equivalently 
interpreted as iterative application of Lemma 3.

To proceed, we use induction in $k$ to show that whenever $\mathcal{G}^{+}[\ldots]$ is acyclic, the required permutation ensuring the disjointness of lines with convex hulls of sets of preceding points exists. To this end, observe first that our statement trivialises for $k=1$. Next, choose a collection $\left(l_{1}, x_{1} ; \ldots ; l_{k}, x_{k}\right)$ and note that if there exists a line in the collection which does not hit the convex hull generated by all the remaining points then by Lemma 3 we can resort to the inductive hypothesis for the collection with the line removed. Consequently, we only have to show that if the collection $\left(l_{1}, x_{1} ; \ldots ; l_{k}, x_{k}\right)$ is such that each line $l_{i}$ hits the convex hull $\operatorname{conv}\left(\left\{x_{j}, j \neq i\right\}\right)$ then $\mathcal{G}^{+}\left[l_{1}, x_{1} ; \ldots ; l_{k}, x_{k}\right]$ contains a cycle. Again resorting to inductive argument if needed, we may assume without loss of generality that $\left(l_{1}, x_{1} ; \ldots ; l_{k}, x_{k}\right)$ is minimal with this property, that is to say it does not contain a proper subcollection such that each its line hits the convex hull generated by the remaining points. This minimality assumption implies that all $x_{i}$ 's are extreme points (vertices) of the convex hull conv $\left(\left\{x_{1}, \ldots, x_{k}\right\}\right)$, for otherwise removing a non-extreme point we would obtain a subcollection enjoying the considered property. For formal convenience we let the vertices $x_{i}$ be ordered clockwise along the boundary of the convex hull and we interpret the indices modulo $k$, that is to say $x_{k+1}=x_{1}$ etc. By minimality, should we remove a vertex $x_{i}$ from the collection, there exists $x_{j}, j \neq i$, such that $l_{j}$ does not hit $\operatorname{conv}\left(\left\{x_{m}, m \neq i, j\right\}\right)$. However, by the assumed properties, the only possible choices for such $j$ are $j=i+\varepsilon$ for $\varepsilon=+1$ or $\varepsilon=-1$ and the line $l_{j}$ has to cross the segment $\overline{x_{i} x_{i-\varepsilon}}$. Consequently, each vertex $x_{i}$ is cut off from the convex hull conv $\left(\left\{x_{1}, \ldots, x_{k}\right\}\right)$ by a line passing through its neighbouring vertex and crossing the opposite neighbouring edge. The choices of $\varepsilon$ for different vertices are not independent, because if we choose to use $l_{i}$ to cut off $x_{i+1}$ then $x_{i-1}$ has to be cut off by $l_{i-2}$. Thus, if the number $k$ of vertices is odd, this is easily checked to imply that either for all $i=1, \ldots, k$ the line $l_{i}$ passes through the segment $\overline{x_{i} x_{i+1}}$ or for all $i=1, \ldots, k$ the line $l_{i}$ passes through the segment $\overline{x_{i} x_{i-1}}$. In both cases this generates a cycle of order $k$ in $\mathcal{G}^{+}\left[l_{1}, x_{1} ; \ldots ; l_{k}, x_{k}\right]$. On the other hand, if $k$ is even, apart from the above two options generating a cycle of order $k$ we have another possibility where the collection of vertices splits into $k / 2$ pairs of neighbours $x_{i}, x_{i+1}$ such that $l_{i}$ crosses $\overline{x_{i+1} x_{i+2}}$ and $l_{i+1}$ crosses $\overline{x_{i-1} x_{i}}$. In this case, however, each such pair of neighbours is contained in the trap region generated by any other one and hence we get a cycle of order 4 consisting of some $x_{i}, y_{i, i+1}, x_{j}, y_{j, j+1}$, where $y_{i, i+1}$ is the intersection point of $l_{i}$ and $l_{i+1}$ and likewise for $y_{j, j+1}$. In either situation $\mathcal{G}^{+}\left[l_{1}, x_{1} ; \ldots ; l_{k}, x_{k}\right]$ contains a cycle as required. This completes the proof of Theorem 5 . 


\subsection{Full characterisation of collective factorisation for rectangu- lar fields}

As we already have mentioned above, in the particular case of rectangular Markov fields our knowledge is more complete than in the general setting. In fact, we are able to show that the necessary precedence graph acyclity condition for collective factorisation on all scales, as stated in combined Lemmas 1 and 2, becomes in this context a sufficient condition as well. This is made precise in the following theorem.

Theorem 6 Assume that the field $\mathcal{A}^{\mathcal{M}}$ is rectangular and let $\left(l_{i}, x_{i}\right)_{i=1}^{k}, x_{i} \in l_{i}$, be a collection of pairwise distinct lines and points in general position. Then the following are equivalent:

1. The precedence graph $\mathcal{G}\left[l_{1}, x_{1} ; \ldots ; l_{k}, x_{k}\right]$ is acyclic.

2. The collection $\left(l_{i}, x_{i}\right)$ admits collective factorisation of correlations on all scales.

Proof The implication from 2. to 1 . follows directly from Lemmas 1 and 2. Thus, it only remains to establish the implication from 1 . to 2 . To this end, we make first the following observation, which is also of its own intrinsic interest in the context of rectangular fields.

Given two non-parallel lines $l$ and $l^{\prime}$ on whose parallel translates the activity measure $\mathcal{M}$ is concentrated, we say that a set $D \subseteq \mathbb{R}^{2}$ is $\left\{l, l^{\prime}\right\}$-convex if for any two points $x, y \in D$ lying on a common translate of $l$ or $l^{\prime}$ the entire segment $\overline{x y}$ is contained in $D$. Clearly, $\left\{l, l^{\prime}\right\}$ convexity is a much weaker concept than the usual convexity, in particular a $\left\{l, l^{\prime}\right\}$-convex set does not even have to be connected. We shall write $\operatorname{conv}_{\left\{l, l^{\prime}\right\}}(A)$ for the $\left\{l, l^{\prime}\right\}$-convex hull of $A \subseteq \mathbb{R}^{2}$, that is to say the smallest $\left\{l, l^{\prime}\right\}$-convex set containing $A$. Now, the crucial observation is that in the context of the generalised dynamic representation in Section 4 specialised for the considered rectangular field, the convexity assumption imposed on the growing family $\left(D_{t}\right)$ there can be relaxed to the $\left\{l, l^{\prime}\right\}$-convexity here without any further modifications of the theory. Moreover, instead of having the initial set $D_{0}$ consist of a single point, we can now let it consist of any positive finite number of points under the $\left\{l, l^{\prime}\right\}$ convexity requirement. Indeed, the proof of Theorem 3 carries over verbatim under these relaxed conditions on $\left(D_{t}\right)$ and the $\left\{l, l^{\prime}\right\}$-convexity of $\left(D_{t}\right)$ ensures that the intersections of 
the field lines with $\left(D_{t}\right)$ are always connected. It is essential to note at this point that the convexity requirement cannot be lifted for the target domain $D$ though. The point is that the consistency statement in Theorem 2 does essentially require convexity of the domain of the field in its proof and, consequently, a rectangular field constructed in some nonconvex but $\left\{l, l^{\prime}\right\}$-convex set may fail to coincide with the corresponding restriction of the whole-plane consistent field. This phenomenon is closely related to the rather unintuitive fact that there exist pairs of compacts $D_{1} \subseteq D_{2} \subseteq \mathbb{R}^{2}$ consisting of a $\left\{l, l^{\prime}\right\}$-convex set $D_{1}$ and a convex set $D_{2}$ with the property that $D_{1}$ cannot be extended to $D_{2}$ through a Hausdorff-continuous growing family of $\left\{l, l^{\prime}\right\}$-convex compacts, in sharp contrast to the case of convex sets where such an extension is always possible. These issues are not discussed in further detail here as falling beyond the scope of the present article, yet they are a subject of our active research in progress because we believe that the knowledge of the geometry of such non-extensible sets and their corresponding defective fields may shed some further light upon the higher-order correlation structure of $\mathcal{A}^{\mathcal{M}}$.

To proceed, recall that the vertex set of the precedence graph $\mathcal{G}\left[l_{1}, x_{1} ; \ldots ; l_{k}, x_{k}\right]$ consists of the generating points $x_{i}$ 's and of $y_{i, j}$ 's arising as the intersection points of the corresponding $l_{i}$ and $l_{j}$. Since the precedence graph is acyclic, there exists a complete ordering of its vertices compatible with the structural partial order induced by directions of its edges. In addition, in view of the minimality of $x_{i}$ 's in the structural order, we can assume that no $y$.,--vertex (intersection vertex) precedes an $x$--vertex (generating vertex) in the considered complete ordering, that is to say the ordered collection of vertices can be written as

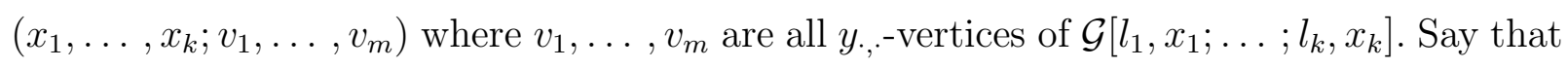
a vertex $u$ of the precedence graph is a direct predecessor of another vertex $w$ if there is a directed edge from $u$ to $w$ containing no other vertices of the graph, which amounts to direct precedence of $u$ over $w$ in the structural order on the vertices of the precedence graph. Note that $y_{\text {., }}$-vertices have precisely two direct predecessors each, whereas $x$.-vertices have no predecessors. We construct inductively a growing family $\left(\tilde{D}_{t}\right)$ of $\left\{l, l^{\prime}\right\}$-convex sets in $\mathbb{R}^{2}$ by putting $\tilde{D}_{t}:=\operatorname{conv}_{\left\{l, l^{\prime}\right\}} D_{t}^{\circ}$, where

- We put $D_{0}^{\circ}=\left\{x_{1}, \ldots, x_{k}\right\}$.

- For $t=i / m, 1 \leq i \leq m$ we set $D_{t}^{\circ}=\left\{x_{1}, \ldots, x_{k}, v_{1}, \ldots, v_{i}\right\}$.

- For $t \in((i-1) / m, i / m), 1 \leq i \leq m$ we let $w_{1}, w_{2}$ be the direct predecessors of the vertex $v_{i}$ (note that $w_{1}, w_{2} \in D_{t}^{\circ}$ for $t \leq(i-1) / m$ by the construction) and we define 
$D_{t}^{\circ}$ as the union of

$-D_{(i-1) / m}^{\circ}$

- the point $w_{1}+m(t-(i-1) / m)\left(v_{i}-w_{1}\right)$,

- the point $w_{2}+m(t-(i-1) / m)\left(v_{i}-w_{2}\right)$.

In other words, initially the set $\tilde{D}_{0}$ consists of $\left\{x_{1}, \ldots, x_{k}\right\}$. Since the collection $\left(l_{i}, x_{i}\right)$ is in general position, we clearly have $\tilde{D}_{0}=\operatorname{conv}_{\left\{l, l^{\prime}\right\}} D_{0}^{\circ}=\operatorname{conv}_{\left\{l, l^{\prime}\right\}}\left\{x_{1}, \ldots, x_{k}\right\}=\left\{x_{1}, \ldots, x_{k}\right\}$. Next, the intersection points $v_{i}$ are subsequently added to the set $D_{t}^{\circ}$, in time intervals of length $1 / \mathrm{m}$. With the intersection points added, the $\left\{l, l^{\prime}\right\}$-convex hull of the increasing vertex collection does no more coincide with the collection itself. The time between the

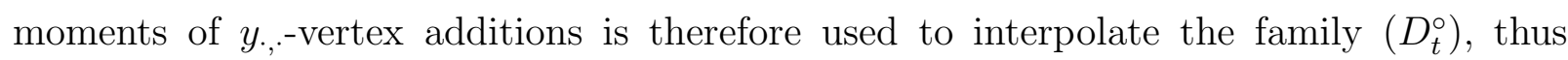
keeping the growth of its $\left\{l, l^{\prime}\right\}$-convex hull $\left(\tilde{D}_{t}\right)$ Hausdorff continuous. Indeed, the growth of $\left(D_{t}^{\circ}\right)$ is Hausdorff continuous by definition and it is easily seen that each new point in $\tilde{D}_{t+d t} \backslash \tilde{D}_{t}$ arises as a shift of a point in $D_{t+d t}^{\circ} \backslash D_{t}^{\circ}$ along a translate of either $l$ or $l^{\prime}$ and, consequently, $\tilde{D}_{t+d t} \backslash \tilde{D}_{t}$ is a union of two rectangles of infinitesimal width $O(d t)$ built on two possibly degenerated segments parallel to $l$ and $l^{\prime}$ respectively. Note that in general this would not be the case if $v_{i}$ 's were not ordered compatibly with the structural ordering - adding a single new intersection point to $D_{t}^{\circ}$ might then possibly result in some further intersection points, not yet present in $D_{t}^{\circ}$, falling into its $\left\{l, l^{\prime}\right\}$-convex hull $\tilde{D}_{t}$, which might in its turn produce an extra non-degenarate rectangle in $\tilde{D}_{t}$ thus violating the Hausdorff continuity. The importance of imposing on $v_{i}$ 's an ordering compatible with the structural order for $\left(l_{i}, x_{i}\right)_{i=1}^{k}$ is that it ensures that the intersection points $v_{i}$ in $\tilde{D}_{t}$ are precisely those present in $D_{t}^{\circ}$ with no extras, that is to say for $t \in[i / m,(i+1) / m) \cap[0,1], i=0,1, \ldots, m$, we have

$$
\left\{x_{1}, \ldots, x_{k}, v_{1}, \ldots, v_{m}\right\} \cap \tilde{D}_{t}=\left\{x_{1}, \ldots, x_{k}, v_{1}, \ldots, v_{i}\right\} \cap D_{t}^{\circ}=\left\{x_{1}, \ldots, x_{k}, v_{1}, \ldots, v_{i}\right\}
$$

In particular, the time order in which the points of the precedence graph $\mathcal{G}\left[l_{1}, x_{1} ; \ldots ; l_{k}, x_{k}\right]$ show up in $\tilde{D}_{t}$ is compatible with the structural ordering for the collection $\left(l_{i}, x_{i}\right)_{i=1}^{k}$. Observe as well that $\tilde{D}_{1}$ is a convex set (a rectangle in fact) containing all $x_{i}$ 's for $i=$ $1, \ldots, k$. Moreover, even though $\left(\tilde{D}_{t}\right)$ does not satisfy the condition (D4) in Section 4 , it can be easily modified by local smoothing to yield an increasing family $\left(D_{t}\right)$ of $\left\{l, l^{\prime}\right\}$ convex sets satisfying the conditions (D1),(D3),(D4),(D5) in Section 4 and enjoying the 
property (inherited from $\left(\tilde{D}_{t}\right)$ ) that $\mathbb{A}\left(l_{i}\right)=x_{i}$ where $\mathbb{A}(\cdot)$ is the anchor mapping induced by $D_{t}$. Furthermore, by the same local smoothing modification $D_{1}$ can be taken convex and containing all $x_{i}$ 's in their interior. In view of the discussion on the particular form of the generalised graphical construction for rectangular fields and taking into account the (GE:LineBirth) rule of this construction we conclude the required collective factorisation of correlations on all scales, thus completing the proof of Theorem 6 .

It should be emphasised at this point that the above argument apparently cannot be repeated for more general fields. Indeed, with all directions allowed the condition (D2) cannot be lifted and $D_{0}$ has to be a singleton which makes our construction above break down. Moreover, in case when only a finite number of, but more than two, directions are allowed, the construction breaks down as well - even though a discussion parallel to the above can be provided based on the concept of convexity in the directions of the field, which does again allow to relax the offending condition (D2), we may in general loose the Hausdorff continuity of $\tilde{D}_{t}$ at the moments when two parts of $\tilde{D}_{t}$ growing in two different directions become for the first time connectable along some other direction of the field (never to happen when there are only two directions allowed).

\subsection{Integral correlation measures}

With the knowledge of the correlation structure of the field $\mathcal{A}^{\mathcal{M}}$ as provided so far in this section, we are now in a position to determine natural integral correlation measures for the field. To this end, for a bounded open convex $D \subseteq \mathbb{R}^{2}$ and for a planar graph in $D$ (a collection of edges, not necessarily in $\Gamma_{D}$ ) we construct its first order directional measure

$$
\mathcal{D}^{(1)}[\gamma]:=\sum_{e \in \operatorname{Edges}(\gamma)} \ell(e) \delta_{l[e]}
$$

with $\ell(\cdot)$ denoting the usual Euclidean length and with $\delta_{l[e]}$ standing for the unit mass at $l[e] \in[[D]]$. The so-defined $\mathcal{D}^{(1)}(\gamma)$ is a finite purely atomic measure on $[[D]]$. Likewise, we consider the second order directional measure on $[[D]] \times[[D]]$ given by

$$
\mathcal{D}^{(2)}[\gamma]:=\sum_{e_{1} \in \operatorname{Edges}(\gamma)} \sum_{e_{2} \in \operatorname{Edges}(\gamma)} \ell\left(e_{1}\right) \ell\left(e_{2}\right) \delta_{\left(l\left[e_{1}\right], l\left[e_{2}\right]\right)} .
$$

We shall use the expectations of these measures with $\gamma$ drawn from $\mathcal{A}_{D}^{\mathcal{M}}$ as natural characteristics of the directional nature of the field. To this end, we put

$$
\Delta_{D}^{\mathcal{M}}:=\mathbb{E} \mathcal{D}^{(1)}\left[\mathcal{A}_{D}^{\mathcal{M}}\right]
$$


Write also

$$
\Sigma_{D}^{\mathcal{M}}:=\mathbb{E} \mathcal{D}^{(2)}\left[\mathcal{A}_{D}^{\mathcal{M}}\right]-\Delta^{\mathcal{M}} \otimes \Delta^{\mathcal{M}}
$$

where $\otimes$ denotes the measure producting operation. Note that $\Sigma_{D}^{\mathcal{M}}$ has its natural interpretation as the directional covariance measure. Below we explicitly determine both the first and second order directional measures for $\mathcal{A}^{\mathcal{M}}$.

Corollary 1 For $l \in[[D]]$ we have

$$
\Delta_{D}^{\mathcal{M}}(d l)=\ell(l \cap D) \mathcal{M}(d l) .
$$

Proof For each $l \in[[D]]$ we have

$$
\Delta^{\mathcal{M}}(d l)=\int_{l \cap D} \sigma^{\mathcal{M}}[d l, x] \ell(d x)
$$

where $\ell$ is the length element. Our statement follows now by Theorem 4 .

Corollary 2 For $l_{1}, l_{2} \in[[D]]$ we have

$$
\Sigma_{D}^{\mathcal{M}}\left(d l_{1}, d l_{2}\right)= \begin{cases}\int_{l \cap D} \int_{l \cap D} \exp (-2 \mathcal{M}([[\overline{x y}]])) \ell(d x) \ell(d y) \mathcal{M}(d l), & \text { if } l_{1}=l_{2}=l, \\ 0, & \text { otherwise }\end{cases}
$$

Proof For $l_{1}, l_{2} \in[[D]]$ we have

$$
\Sigma_{D}^{\mathcal{M}}\left(d l_{1}, d l_{2}\right)=\int_{l_{1} \cap D} \int_{l_{2} \cap D} \sigma^{\mathcal{M}}\left[d l_{1}, x_{1} ; d l_{2}, x_{2}\right] \ell\left(d x_{2}\right) \ell\left(d x_{1}\right)-\Delta_{D}^{\mathcal{M}}\left(d l_{1}\right) \Delta_{D}^{\mathcal{M}}\left(d l_{2}\right) .
$$

Our assertion follows now by Theorem 4 .

\subsection{Alternative correlation measures}

In addition to the events that there exist field lines passing through given points in given directions as considered in the definition of $\sigma^{\mathcal{M}}[\ldots]$ above, one can also study events that there exist field angles with vertices at given points and with their arms extending along given half-lines. In formal terms, we put

$$
\varsigma^{\mathcal{M}}\left[d \angle_{1} ; \ldots ; d \angle_{k}\right]:=d \mathbb{P}\left(\forall_{i=1}^{k} \angle_{k} \in \operatorname{Angles}\left(\mathcal{A}^{\mathcal{M}}\right)\right),
$$

where Angles $(\gamma), \gamma \in \Gamma$, denotes the collection of convex angles in the admissible polygonal graph $\gamma$ and where $L_{i}:=\angle\left[\vec{l}_{i}, \vec{l}_{i}{ }_{i}\right], i=1, \ldots, k$, stands for the convex angle between two 
directed half-lines $\vec{l}_{i}$ and ${\overrightarrow{l^{\prime}}}_{i}$ outgoing from the vertex of the angle and directed away from the vertex. It should be noted at this point for formal completeness that the concave angles arising as the complements of the convex angles of $\gamma$ are not included into Angles $(\gamma)$. In analogy to Lemma 3, a similar factorisation result for the probabilities of such events can be concluded from the generalised dynamic representation.

Corollary 3 Assume the collection $\angle_{1}, \ldots, \angle_{k}$ is such that the vertices $x_{i}$ of $\angle_{i}$ are all different and such that the interior of the convex angle $L_{1}$ is disjoint with the convex hull $\operatorname{conv}\left(\left\{x_{2}, \ldots, x_{k}\right\}\right)$. Then

$$
\varsigma^{\mathcal{M}}\left[d \angle_{1} ; \ldots ; d \angle_{k}\right]=\mathcal{M}\left(d l_{1}\right) \mathcal{M}\left(d l_{1}^{\prime}\right) \varsigma^{\mathcal{M}}\left[d \angle_{2} ; \ldots ; d \angle_{k}\right] .
$$

Proof Indeed, under the assumptions of the corollary the increasing family $D_{t}$ in the generalised dynamic representation in Section 4 can be chosen so that it first hits all $x_{2}, \ldots, x_{k}$ and finally it reaches $x_{1}$ before hitting any other point of $\angle_{1}$. Our claim follows now by the rule (GE:VertexBirth) of the generalised representation.

Suppose now that we assign alternating labels +1 and -1 to regions separated by the contours of the field which thus become interfaces between +1 and -1 phases. This gives rise to two possible label assignments in $\mathbb{R}^{2}$ and we pick any of them with probability $1 / 2$. Write $\left[\mathcal{A}^{\mathcal{M}}\right]_{x}$ for the label assigned by $\mathcal{A}^{\mathcal{M}}$ at $x \in \mathbb{R}^{2}$. Then for two points $x, y \in \mathbb{R}^{2}$ we can define the two-point label-correlation function

$$
\rho_{x, y}^{\mathcal{M}}:=\mathbb{E}\left[\mathcal{A}^{\mathcal{M}}\right]_{x}\left[\mathcal{A}^{\mathcal{M}}\right]_{y}-\mathbb{E}\left[\mathcal{A}^{\mathcal{M}}\right]_{x} \mathbb{E}\left[\mathcal{A}^{\mathcal{M}}\right]_{y}
$$

It is easily seen that, writing $n_{x, y}:=\operatorname{card}\left(\overline{x y} \cap \mathcal{A}^{\mathcal{M}}\right)$ for the number of edges of the field $\mathcal{A}^{\mathcal{M}}$ crossing the segment $\overline{x y}$,

$$
\rho_{x, y}^{\mathcal{M}}=\mathbb{P}\left(n_{x, y} \text { is even }\right)-\mathbb{P}\left(n_{x, y} \text { is odd }\right) .
$$

Recalling from the Linear sections statement of Theorem 2 that $n_{x, y}$ is Poisson with parameter $\mathcal{M}([[\overline{x y}]])$ with $\overline{x y}$ denoting the segment joining $x$ to $y$, we conclude that

Corollary 4 We have

$$
\rho_{x, y}^{\mathcal{M}}=\exp (-\mathcal{M}([[\overline{x y}]])) .
$$

Roughly speaking, this shows that for regularly behaved activity measures $\mathcal{M}$ the twopoint label-correlation functions of the field $\mathcal{A}^{\mathcal{M}}$ exhibit exponential decay with the distance between $x$ and $y$ which places the inverse temperature $\beta=1$ corresponding to the consistent regime in the high temperature regime for polygonal fields, above the phase transition point. 


\section{Disagreement loop death and birth dynamics}

In this section we discuss a random dynamics on the space $\Gamma_{D}$ of admissible polygonal configurations which leaves the law of the consistent field $\mathcal{A}_{D}^{\mathcal{M}}$ invariant, with $D$ standing as usual for an open bounded convex set in $\mathbb{R}^{2}$. We also provide a modification of this dynamics which can be used for Monte-Carlo simulation of $\mathcal{A}_{D}^{\mathcal{M} ; \beta}$ for all $\beta \in \mathbb{R}$. We build upon [15] in our presentation of the standard dynamics based on an important concept of a disagreement loop.

\subsection{Standard disagreement loop dynamics for consistent regime}

To proceed we place ourselves within the context of the standard dynamic representation as given in Subsection 3.1 and suppose that we observe a particular realisation $\gamma \in \Gamma_{D}$ of the polygonal field $\mathcal{A}_{D}^{\mathcal{M}}$ and that we modify the configuration by adding an extra birth site $x_{0}$ to the existing collection of birth sites for $\gamma$, while keeping the evolution rules (E1-4) for all the particles, including the two newly added ones if $x_{0} \in D$ and the single newly added one if $x_{0} \in \partial D$. Denote the resulting new (random) polygonal configuration by $\gamma \oplus x_{0}$. A simple yet crucial observation is that for $x_{0} \in D$ the symmetric difference $\gamma \triangle\left[\gamma \oplus x_{0}\right]$ is almost surely a single loop (a closed polygonal curve), possibly self-intersecting and possibly chopped off by the boundary. Indeed, this is seen as follows. The leftmost point of the loop $\gamma \triangle\left[\gamma \oplus x_{0}\right]$ is of course $x_{0}$. Each of the two new particles $p_{1}, p_{2}$ emitted from $x_{0}$ move independently, according to $(\mathbf{E} 1-\mathbf{4})$, each giving rise to a disagreement path. The initial segments of such a disagreement path correspond to the movement of a particle, say $p_{1}$, before its annihilation in the first collision. If this is a collision with the boundary, the disagreement path gets chopped off and terminates there. If this is a collision with a segment of the original configuration $\gamma$ corresponding to a certain old particle $p_{3}$, the new particle $p_{1}$ dies but the disagreement path continues along the part of the trajectory of $p_{3}$ which is contained in $\gamma$ but not in $\gamma \oplus x_{0}$. At some further moment $p_{3}$ dies itself in $\gamma$, touching the boundary or killing another particle $p_{4}$ in $\gamma$. In the second case, however, this collision only happens for $\gamma$ and not for $\gamma \oplus x_{0}$ so the particle $p_{4}$ survives (for some time) in $\gamma \oplus x_{0}$ yielding a further connected portion of the disagreement path initiated by $p_{1}$, which is contained in $\gamma \oplus x_{0}$ but not in $\gamma$ etc. A recursive continuation of this construction shows that the disagreement path initiated by $p_{1}$ consists alternately of connected polygonal subpaths contained in $\left[\gamma \oplus x_{0}\right] \backslash \gamma$ (call these creation phase subpaths) 
and in $\gamma \backslash\left[\gamma \oplus x_{0}\right]$ (call these annihilation phase subpaths). Note that this disagreement path is self-avoiding and, in fact, it can be represented as the graph of some piecewise linear function $t \mapsto y(t)$. Clearly, the same applies for the disagreement path initiated by $p_{2}$. An important observation is that whenever two creation phase or two annihilation phase subpaths of the two disagreement paths hit each other, both disagreement paths die at this point and the disagreement loop closes (as opposed to intersections of segments of different phases which do not have this effect). Obviously, if the disagreement loop does not close in the above way, it gets eventually chopped off by the boundary. We shall write $\Delta^{\oplus}\left[x_{0} ; \gamma\right]=\gamma \Delta\left[\gamma \oplus x_{0}\right]$ to denote the (random) disagreement loop constructed above. It remains to consider the case $x_{0} \in \partial D$, which is much simpler because there is only one particle emitted and so $\Delta^{\oplus}\left[x_{0} ; \gamma\right]=\gamma \triangle\left[\gamma \oplus x_{0}\right]$ is a single self-avoiding polygonal path eventually chopped off by the boundary. We shall often abuse the language calling such a disagreement path $\Delta^{\oplus}\left[x_{0} ; \gamma\right]$ a (degenerate) disagreement loop as well.

Likewise, a disagreement loop (or path) arises if we remove one birth site $x_{0}$ from the collection of birth sites of an admissible polygonal configuration $\gamma \in \Gamma_{D}$, while keeping the evolution rules for all the remaining particles. We write $\gamma \ominus x_{0}$ for the configuration obtained from $\gamma$ by removing $x_{0}$ from the list of the birth sites, while the resulting random disagreement loop (or path) is denoted by $\Delta^{\ominus}\left[x_{0} ; \gamma\right]$ so that $\Delta^{\ominus}\left[x_{0} ; \gamma\right]=\gamma \triangle\left[\gamma \ominus x_{0}\right]$. We refer the reader to Section 2.1 in [15] for further discussion.

With the above terminology we are in a position to describe a random dynamics on the configuration space $\Gamma_{D}$, which leaves invariant the law of the consistent polygonal process $\mathcal{A}_{D}^{\mathcal{M}}$. Particular care is needed, however, to distinguish between the notion of time considered in the dynamic representation of the field as well as throughout the construction of the disagreement loops above, and the notion of time to be introduced for the random dynamics on $\Gamma_{D}$ constructed below. To make this distinction clear we shall refer to the former as to the representation time (r-time for short) and shall reserve for it the notation $t$, while the latter will be called the simulation time (s-time for short) and will be consequently denoted by $s$ in the sequel.

Consider the following pure jump birth and death type Markovian dynamics on $\Gamma_{D}$, with $\gamma_{s}=\gamma_{s}^{D}$ standing for the current configuration

DL:birth With intensity $\langle\langle\mathcal{M}\rangle\rangle(d x) d s$ for $x \in D$ and with intensity $\langle\langle\mathcal{M} ; \partial D\rangle\rangle(d x) d s$ for $x \in \partial D$ set $\gamma_{s+d s}:=\gamma_{s} \oplus x$, 
DL:death For each birth site $x$ in $\gamma_{s}$ with intensity $d s$ set $\gamma_{s+d s}:=\gamma_{s} \ominus x$.

If none of the above updates occurs we keep $\gamma_{s+d s}=\gamma_{s}$. It is convenient to perceive the above dynamics in terms of generating random disagreement loops $\lambda$ and setting $\gamma_{s+d s}:=$ $\gamma_{s} \Delta \lambda$, with the loops of the type $\Delta^{\oplus}[\cdot, \cdot]$ corresponding to the rule DL:birth and $\Delta^{\ominus}[\cdot, \cdot]$ to the rule DL:death.

As a direct consequence of the dynamic representation of the consistent field $\mathcal{A}_{D}^{\mathcal{M}}$ we obtain

Theorem 7 The distribution of the polygonal field $\mathcal{A}_{D}^{\mathcal{M}}$ is the unique invariant law of the dynamics given by DL:birth and DL:death. The resulting s-time stationary process is reversible. Moreover, for any initial distribution of $\gamma_{0}$ the laws of the polygonal fields $\gamma_{s}$ converge in variational distance to the law of $\mathcal{A}_{D}^{\mathcal{M}}$ as $s \rightarrow \infty$.

The uniqueness and convergence statements in the above theorem require a short justification. They both follow by the observation that, in finite volume, regardless of the initial state, the process $\gamma_{s}$ spends a non-null fraction of time in the empty state (no polygonal contours). Indeed, this observation allows us to conclude the required uniqueness and convergence by a standard coupling argument, e.g. along the lines of the proof of Theorem 1.2 in [13].

\subsection{Standard disagreement loop dynamics for general tempera- tures}

Below, we show that the laws of the Gibbs-modified polygonal fields $\mathcal{A}_{D}^{\mathcal{M} ; \beta}, \beta \in \mathbb{R}$, arise as the unique invariant distributions for appropriate modifications of the reference dynamics DL:birth, DL:death. The main change is that the birth and death updates are no more performed unconditionally, they pass an acceptance test instead and are accepted with certain state-dependent probabilities whereas upon failure of the acceptance test the update is discarded. Consider the following dynamics

DL : $\operatorname{birth}[\beta]$ With intensity $\langle\langle\mathcal{M}\rangle\rangle(d x) d s$ for $x \in D$ and with intensity $\langle\langle\mathcal{M} ; \partial D\rangle\rangle(d x) d s$ for $x \in \partial D$ do

- $\operatorname{put} \delta:=\gamma_{s} \oplus x$

- accept $\delta$ with probability $\exp \left(-[\beta-1]\left(L^{\mathcal{M}}(\delta)-L^{\mathcal{M}}\left(\gamma_{s}\right)\right)\right)$ if $L^{\mathcal{M}}(\delta)>L^{\mathcal{M}}\left(\gamma_{s}\right)$ and with probability 1 otherwise, 
- if accepted, set $\gamma_{s+d s}:=\delta$, otherwise keep $\gamma_{s+d s}:=\gamma_{s}$.

DL : $\operatorname{death}[\beta]$ For each birth site $x$ in $\gamma_{s}$ with intensity $d s$ do

- $\operatorname{put} \delta:=\gamma_{s} \ominus x$,

- accept $\delta$ with probability $\exp \left(-[\beta-1]\left(L^{\mathcal{M}}(\delta)-L^{\mathcal{M}}\left(\gamma_{s}\right)\right)\right)$ if $L^{\mathcal{M}}(\delta)>L^{\mathcal{M}}\left(\gamma_{s}\right)$ and with probability 1 otherwise,

- if accepted, set $\gamma_{s+d s}:=\delta$, otherwise keep $\gamma_{s+d s}:=\gamma_{s}$.

In analogy with its original reference form DL:birth, DL:death, the above dynamics should be thought of as generating random disagreement loops $\lambda$ and setting $\gamma_{s+d s}:=$ $\gamma \triangle \lambda$ provided $\lambda$ passes the acceptance test. The following theorem justifies the above construction.

Theorem 8 For each $\beta \in \mathbb{R}$ the law of the polygonal process $\mathcal{A}_{D}^{\mathcal{M} ; \beta}$ is the unique invariant distribution of the dynamics $\mathbf{D L}: \operatorname{birth}[\beta], \mathbf{D L}: \operatorname{death}[\beta]$. The resulting s-time stationary process is reversible. For any initial distribution of $\gamma_{0}$ the laws of the polygonal fields $\gamma_{s}$ converge in variational distance to the law of $\mathcal{A}_{D}^{\mathcal{M} ; \beta}$ as $s \rightarrow \infty$.

Indeed, the invariance follows by a straightforward check of usual detailed balance conditions whereas the convergence and uniqueness statements are verified as in Theorem 7.

\subsection{Generalised disagreement loop dynamics}

Even though the above disagreement loop dynamics in its standard version has been constructed using the standard dynamic representation of Subsection 3.1, exactly the same can be made for the generalised dynamic representation of Section 4. As easily verified, this also leads to disagreement loop and path creation and annihilation, the only difference being that boundary birth events in the standard set-up are replaced by general line birth events in context of the generalised dynamic construction. Vertex creation and annihilation yield disagreement loops whereas line creation and annihilation yield disagreement paths. Thus, the DL:birth and DL:death moves get replaced by

GenDL:birth With intensity $\langle\langle\mathcal{M}\rangle\rangle(d x) d s$ for $x \in D$ set $\gamma_{s+d s}:=\gamma_{s} \oplus x$. With intensity $\mathcal{M}(d l) d s$ for $l \in[[D]]$ set $\gamma_{s+d s}:=\gamma_{s} \oplus l$, where $\gamma_{s} \oplus l$ arises from $\gamma_{s}$ by letting $l$ 
be born at its anchor point $\mathbb{A}(l)$ and making it extend thereupon according to the evolution rules of the generalised construction, which gives rise to a disagreement path.

GenDL:death For each point birth site $x$ in $\gamma_{s}$ with intensity $d s$ set $\gamma_{s+d s}:=\gamma_{s} \ominus x$. For each line birth site $\mathbb{A}(l)$ of a line $l$ in $\gamma_{s}$, with intensity $d s$ set $\gamma_{s+d s}:=\gamma_{s} \ominus l$, where again $\gamma_{s} \ominus l$ arises from $\gamma_{s}$ by killing at $\mathbb{A}(l)$ the field line $l$ of $\gamma_{s}$, which gives rise to a disagreement path.

In full analogy with Theorem 7 we have

Theorem 9 The distribution of the polygonal field $\mathcal{A}_{D}^{\mathcal{M}}$ is the unique invariant law of the dynamics given by GenDL:birth and GenDL:death. The resulting s-time stationary process is reversible. Moreover, for any initial distribution of $\gamma_{0}$ the laws of the polygonal fields $\gamma_{s}$ converge in variational distance to the law of $\mathcal{A}_{D}^{\mathcal{M}}$ as $s \rightarrow \infty$.

The modification GenDL $[\beta]$ of the GenDL dynamics for general $\beta \in \mathbb{R}$ goes along exactly the same lines as the construction of $\mathbf{D L}[\beta]$ from $\mathbf{D L}$, by introducing suitable acceptance tests, and hence we omit the standard details here. Obviously, the corresponding version of Theorem 8 holds in full analogy. Clearly, the so-defined dynamics depends then on the choice of the increasing family $D_{t}$. Note that hybrid dynamics can also be considered, switching between different choices of $D_{t}$ in the course of the simulation time $s$, which is sometimes beneficial e.g. in image processing applications, see [17].

\subsection{Defective disagreement loop dynamics}

To conclude the discussion of disagreement loop dynamics we introduce one more variant thereof, which we call defective dynamics. The idea stems from the observation that the standard dynamic construction in Subsection 3.1 and its generalised version in Section 4 rely respectively on the time axis direction and on the anchor mapping $\mathbb{A}:[[D]] \rightarrow D$ to decide in which of the two possible directions to proceed along a new line each time a directional update occurs and a new edge is to be created. This decision mechanism is then inherited by the disagreement loop dynamics where the current direction of an unfolding disagreement loop changes in the following situations:

1. upon a directional update during a creation phase, 
2. when a directional update is present on the path being erased during an annihilation phase,

3. when switching between creation and annihilation phases.

In all these situations the choice of the direction to follow along the new line is always the same: forward in time in the standard case and away from the corresponding anchor point in the generalised case. In certain contexts there may be good reasons to try to overcome these restrictions and to allow for more general directional decision strategies even at the cost of obtaining disagreement loop dynamics which do not correspond to any well-defined dynamic representations. One of such situations arises when studying the correlation functions of the polygonal field as defined in Section 5 and we shall indeed use the defective construction to provide below a proof of exponential correlation decay for rectangular fields, see Section 7. Another setting where more general directional decision strategies may be of use are image processing applications of polygonal fields, see [17].

The idea underlying the defective disagreement loop dynamics is hence to keep the crucial feature of the above DL and GenDL dynamics, being that the symmetric difference between a configuration and its update is always a disagreement loop algorithmically constructible in the course of alternating creation and annihilation phases, but to give up the requirement that the disagreement loop generating mechanism be determined by a dynamic representation. Such an approach makes this disagreement loop generating mechanism the core of the construction. In our construction of the defective dynamics we shall assume that upon each directional update along an unfolding disagreement loop, the decision on which of the two possible directions to choose along the new line is taken as a deterministic function of the current portion of the disagreement subpath built by the time of the update. To put it in formal terms, assume we are given a measurable directional decision mapping/rule $\phi=\phi_{D}$ assigning to each connected non-closed but possibly self-intersecting polygonal path $\lambda$ contained in the domain $D$ of the field and to a line $l \in[[D]]$ passing through one of the two ends of $\lambda$, one of the two possible directions along $l$. In our dynamics below $\phi(\lambda, l)$ will be used to determine the direction in which the disagreement path $\lambda$ is to extend upon a directional update into $l$. For a polygonal configuration $\gamma \in \Gamma_{D}$, a straight line $l \in[[D]]$ assumed not to be an extension of an edge of $\gamma$ and a point $x \in l \backslash \gamma$ we construct the configuration $\gamma \oplus(l, x) \in \Gamma_{D}$, interpreted as the result of adding to $\gamma$ a linear germ $(l, x)$ born at $x$ and initially evolving along $l \ni x$ or, in other words, creating a new 
field line for $\gamma$ at $x$. The construction goes as follows. We add a line birth site for $l$ at $x$ at the time $t=0$ whereupon we let the newly born linear germ extend in both directions along $l$ away from $x$, say with constant unit speed for definiteness although other options are also possible as discussed below. While extending, the resulting new edge is subject to directional updates: for each line $l^{\prime}$ which the growing edge crosses the probability that a directional update occurs and a new growth direction along $l^{\prime}$ is assumed is $\mathcal{M}\left(d l^{\prime}\right)$ - if this happens at some time moment $t$, the new direction along $l^{\prime}$ is always chosen according to the directional decision rule $\phi\left(\lambda_{t}, l^{\prime}\right)$ and the growth continues with constant unit speed, with $\lambda_{t}$ standing for the current path constructed by the time $t$. At some point, the resulting polygonal path may hit the boundary of $D$ or an existing edge $e$ of $\gamma$, in which case it stops growing in this direction. Upon a collision with an edge we switch from creation mode to annihilation mode and proceed (say, again with constant unit speed) from the collision point along the edge $e$ in the direction indicated by $\phi\left(\lambda_{t}, l[e]\right)$, erasing this part of the edge. This continues till we reach the boundary $\partial D$ or another edge $e^{\prime}$ of $\gamma$. In the latter case, if the edge $e^{\prime}$ extends in the direction agreeing with $\phi\left(\lambda_{t}, l\left[e^{\prime}\right]\right)$ from the collision point, we proceed along $e^{\prime}$ and keep erasing it. If, however, the edge $e^{\prime}$ extends from the collision point in the direction opposite to $\phi\left(\lambda_{t}, l\left[e^{\prime}\right]\right)$, we switch back to the creation mode and start extending $e^{\prime}$ in the direction indicated by $\phi\left(\lambda_{t}, l\left[e^{\prime}\right]\right)$. We continue this procedure for both branches of the linear germ emitted from the line birth site $(l, x)$. Both branches give rise to directed polygonal disagreement paths constituting the symmetric difference $\gamma \triangle(\gamma \oplus(l, x))$, both consisting of alternating polygonal subpaths corresponding to creation and annihilation stages of the construction and respectively referred to as creation phase and annihilation phase subpaths in the sequel. The procedure terminates when both branches get killed in collision with $\partial D$. It may be useful to observe that the requirement that both branches of the disagreement loop grow with constant unit speed has no effect on the final shape of individual branches but it determines the precedence of their intersection points, should they meet. Thus, in principle, the unit growth speed may be alternatively replaced by other natural options such as letting the right (left) branch grow first till it reaches the boundary and to let the left (right) branch grow thereupon, or even admitting different growth speeds for both branches or speeds depending on the current form of the disagreement loop under construction. Below we shall always assume one of these options is chosen and fixed, under each such choice the discussion below remains valid. The co-existence of two disagreement branches obliges us to impose one more rule 
on the construction of $\gamma \oplus(l, x)$ : if a creation phase subpath of one branch hits a creation phase subpath of another branch, both branches get killed at this point, referred to as the cut-off point below, their continuation possibly already constructed gets chopped off and we consider the disagreement loop complete thus terminating the construction; likewise for two annihilation phase subpaths of different branches colliding. If two subpaths of different phases coming from different branches meet, nothing happens though. Note that there can be several such cut-off points and the disagreement loop construction terminates at the first cut-off point with respect to the ordering imposed by the time flow - consequently the choice of a particular growth speed protocol as discussed above may affect the choice of the loop-closing cut-off point and hence also the final shape of the disagreement loop.

So far, this construction looks very similar to the construction of the disagreement paths and loops in DL and GenDL above. There is one crucial difference though: it may happen here that a self-intersection occurs along one branch of the disagreement path being constructed - this could never have happened in the disagreement loop dynamics DL and GenDL corresponding to dynamic representations because of the temporal ordering along branches of disagreement paths/loops there, and this is the price we pay here for the freedom of choice of the directional decision mapping $\phi(\cdot, \cdot)$. If such a self-intersection occurs past the cut-off point, we just ignore it. If it happens before the cut-off, we are in trouble though. A look at the description of the disagreement path construction procedure here readily shows that the presence of such a self-intersection and hence of a cycle along a single disagreement branch before the cut-off means

- either that at this point we start discarding the changes previously introduced by erasing segments previously created (meeting of two creation phases)

- or that the $\oplus(l, x)$ operation is not reversible (creation phase edge hits annihilation phase subpath preceding it along the disagreement branch) in that the disagreement loop $\gamma \triangle(\gamma \oplus(l, x))$, even though it can be used for the transition $\gamma \rightarrow(\gamma \oplus(l, x))$, is no more a valid disagreement loop upon switching the creation and annihilation phases and thus cannot be used for the reverse transition $(\gamma \oplus(l, x)) \rightarrow \gamma$.

The remaining two situations: intersection of two annihilation subpaths or an annihilation edge hitting a creation subpath preceding it along the disagreement branch, cannot occur in our construction as this would either imply the existence of a node of order higher than 2 in the original configuration $\gamma$ (in the first case) or stand in contradiction to the 
disagreement loop construction rules (in the second case). To prevent the above pathologies from happening we say that the construction of the disagreement loop/path fails if a cycle occurs along one of the branches. Consequently, the configuration $\gamma \oplus(l, x)$ is not always defined, hence the name defective dynamics.

In full analogy with the above definition of the $\oplus$ operation, we can define the configuration $\gamma \ominus(l, x) \in \Gamma_{D}$ for line $l$ extending an edge $e \in \operatorname{Edges}(\gamma), \gamma \in \Gamma_{D}$, with $x \in e$, which is to be interpreted as initiating the annihilation phase in $\gamma$ from the linear germ $(l, x)$ or, in other words, killing/annihilating the field line $l$ of $\gamma$ at the point $x$. This operation also gives rise to a disagreement loop and its outcome may be undefined as well, on equal rights with $\ominus$.

The operations $\oplus(l, x)$ and $\ominus(l, x)$ combine the properties of line birth/death and vertex birth/death operations of the generalised disagreement loop dynamics in Subsection 6.3 and there are no separate $\oplus l, \oplus x, \ominus l, \ominus x$ operations in the defective dynamics.

Directly from the definition of the line creation operation $\oplus$ it follows that if for some $\gamma \in \Gamma_{D}$ and a linear germ $(l, x), x \in l \backslash \gamma$, another configuration $\gamma^{\prime} \in \Gamma_{D}$ is reachable from $\gamma$ upon adding the linear germ $(l, x)$, that is to say $\gamma^{\prime}$ is a possible outcome for $\gamma \oplus(l, x)$, then the probability element that $\gamma \oplus(l, x)=\gamma^{\prime}$ is

$$
\exp \left(-L^{\mathcal{M}}\left(\gamma^{\prime} \backslash \gamma\right)\right) \prod_{e \in \operatorname{Edges}\left(\gamma^{\prime} \backslash \gamma\right), e \not x} \mathcal{M}(d l[e]),
$$

where $\operatorname{Edges}\left(\gamma^{\prime} \backslash \gamma\right)$ stands for the collection of edges of $\gamma^{\prime}$ which are not extensions of edges of $\gamma$ and thus they have to result from directional updates during creation phase. Indeed, the product $\prod \mathcal{M}(d l[e])$ in (19) comes from the probability cost of creation phase directional updates yielding all edges in $\operatorname{Edges}\left(\gamma^{\prime} \backslash \gamma\right)$ save the initial one germinating from $x$, whereas $\exp \left(-L^{\mathcal{M}}\left(\gamma^{\prime} \backslash \gamma\right)\right)$ is due to the absence of directional updates along the created polygonal paths of $\gamma^{\prime} \backslash \gamma$. In a similar way we see that if for some $(l, x)$ the configuration $\gamma \in \Gamma_{D}$ contains an edge $e$ with $x \in e$ and $l=l[e]$ then for another configuration $\gamma^{\prime}$ reachable from $\gamma$ by annihilating the line $l$ at $x$ we have the probability element that $\gamma \ominus(l, x)=\gamma^{\prime}$ given by

$$
\exp \left(-L^{\mathcal{M}}\left(\gamma^{\prime} \backslash \gamma\right)\right) \prod_{e \in \operatorname{Edges}\left(\gamma^{\prime} \backslash \gamma\right)} \mathcal{M}(d l[e]) .
$$

It is also crucial to observe that each valid transition $\gamma \rightarrow \gamma^{\prime}=\gamma \oplus(l, x)$ admits its inverse obtainable as $\gamma^{\prime} \rightarrow \gamma=\gamma^{\prime} \ominus(l, x)$ under the same directional decisions made in the course of 
the disagreement loop construction but with the creation and annihilation phases switched - this is due to the fact that the directional decision rule $\phi(\cdot, \cdot)$ only takes into account the current state of the unfolding disagreement loop (which is the same for $\gamma \rightarrow \gamma^{\prime}$ and $\gamma^{\prime} \rightarrow \gamma$ as the symmetric difference) rather than the actual configuration (which differs for both transitions).

Consider now a measurable set $A_{L} \subseteq[[D]]$ and a measurable mapping $\mathbb{A}^{*}: A_{L} \rightarrow D$ such that $\mathbb{A}^{*}(l) \in l$. These are interpreted respectively as the set of lines allowed for creation/annihilation and the assignment to each line of its germination point. The mapping $\mathbb{A}^{*}$ can also be randomised as long as it is kept independent of the dynamics below, but for simplicity we do not discuss the details of this straightforward extension as it is not going to be used in this paper. Define the following defective disagreement loop dynamics unfolding in s-time:

DefDL:birth With intensity $\mathcal{M}(d l) d s$ for $l \in A_{L}$ set $\gamma_{s+d s}:=\gamma_{s} \oplus\left(l, \mathbb{A}^{*}(l)\right)$. Should the proposed update fail, keep $\gamma_{s+d s}=\gamma_{s}$.

DefDL:death For each line $l \in A_{L}$ extending an edge $e$ of $\gamma_{s}$ such that $\mathbb{A}^{*}(l) \in e$, with intensity $d s$ set $\gamma_{s+d s}:=\gamma_{s} \ominus\left(l, \mathbb{A}^{*}(l)\right)$. Should the proposed update fail, keep $\gamma_{s+d s}=\gamma_{s}$

Recalling the above transition probabibilities (19) and (20) corresponding to $\oplus$ and $\ominus$ moves and taking into account the discussion following their displays allows for a straightforward verification of the detailed balance conditions which readily yields

Theorem 10 The distribution of the polygonal field $\mathcal{A}_{D}^{\mathcal{M}}$ is an invariant law for the dynamics given by DefDL:birth and DefDL:death. The resulting s-time stationary process is reversible.

Note that in strong contrast to analogous Theorems 7 and 9 we do not state uniqueness or convergence to the law of the polygonal field $\mathcal{A}_{D}^{\mathcal{M}}$ here and this is because in general neither of them holds. Indeed, this is due to the fact that a defective dynamics does not have to be transitive: there may exists pairs of admissible polygonal configurations which cannot be reached from each other by $\oplus$ and $\ominus$ moves. This is closely related to the possible failures of these updates and it is the price to pay for the free choice of the directional decision mapping. The structure of the recurrent classes of defective dynamics on $\Gamma_{D}$ is a subject of our research in progress. Observe as well that, in view of the discussion 
following the displays (19) and (20), the requirement that the directional decision rule $\phi(\cdot, \cdot)$ only depends on the current state of the disagreement loop rather than on the entire actual configuration seems indispensable for the detailed balance and the reversibility of the dynamics.

\section{Variants and applications of the defective dynamics The modification DefDL $[\beta]$} of the defective disagreement loop dynamics DefDL for general $\beta \neq 1$ is straightforward and goes by introducing suitable acceptance test for update proposals in full analogy with the corresponding modification of the DL dynamics aimed at obtaining $\mathbf{D L}[\beta]$ in Subsection 6.2 .

The class of possible directional decision rules is extremely rich and various strategies can be chosen encompassing different goals. One possible option of constructing the directional decision mapping goes by choosing an arbitrary measurable generalised anchor mapping $\mathbb{A}:[[D]] \rightarrow D$ with $\mathbb{A}(l) \in l$ to be interpreted as the initial point of the line $l$. Then the direction $\phi(\lambda, l)$ the disagreement loop $\lambda$ is to assume for its unfolding along $l$ upon a directional update is always set away from the anchor point $\mathbb{A}(l)$. Note that if the anchor mapping corresponds to an increasing family $\left(D_{t}\right)$ of convex compacts satisfying (D1-5) as in Section 4 then the resulting defective dynamics $\oplus$ and $\ominus$ operations coincide respectively with the line birth and line death operations of the generalised diasgreement loop dynamics described in Subsection 6.3.

The directional decision mapping can also be randomised, as long as $\phi\left(\lambda_{t}, \cdot\right)$ is independent of the current field configuration conditionally given $\lambda_{t}$. Such randomised strategies will not be used in this paper though.

Due to its flexibility, we envision manifold applications of the defective dynamics in our research in progress on polygonal fields. In the first place we are planning to use it to gain further knowledge on the higher order correlation structure of consistent polygonal fields. To illustrate the techniques we are currently developing we shall employ the defective dynamics to establish exponential decay of correlations in the particular case of rectangular fields in Section 7 below. Another important field in which we anticipate the use of this dynamics is digital image segmentation - the defective dynamics will provide a rich and flexible class of new Monte-Carlo moves for polygonal field-based Bayesian segmentation algorithms we developed in joint work with Kluszczyński and van Lieshout [10, 11, 12, 17]. 


\section{Exponential decay of dependencies for consistent rectangular fields}

The purpose of this section is to make use of the defective disagreement loop dynamics developed in Subsection 6.4 above in order to establish exponential decay of higher order correlations for the consistent rectangular field $\mathcal{A}^{\mathcal{M}}$. To simplify the presentation we assume throughout this section with no loss of generality that the admissible directions $l, l^{\prime}$ of the field are parallel to the coordinate axes. Assume also that a collection $\left(l_{i}, x_{i}\right)_{i=1}^{k}, x_{i} \in l_{i}$, in general position as in Section 5 is given such that $x_{1}$ is its topmost point with the highest $y$ coordinate. Further, let $\mathcal{R}\left[x_{2}, \ldots, x_{k}\right]$ be the smallest rectangle with sides parallel to coordinate axes containing all remaining points $x_{2}, \ldots, x_{k}$ of the considered collection. We know from Lemma 3 that if $l_{1}$ does not hit the convex hull of $x_{2}, \ldots, x_{k}$ then the correlation function $\sigma^{\mathcal{M}}\left[l_{1}, x_{1} ; l_{2}, x_{2} ; \ldots ; l_{k}, x_{k}\right]$ factorises as $\sigma^{\mathcal{M}}\left[l_{1}, x_{1}\right] \sigma^{\mathcal{M}}\left[l_{2}, x_{2} ; \ldots ; l_{k}, x_{k}\right]$. Here in Lemma 4 we show that also when $l_{1}$ does hit $\operatorname{conv}\left(\left\{x_{2}, \ldots, x_{k}\right\}\right)$ and hence it also hits the rectangle $\mathcal{R}\left[x_{2}, \ldots, x_{k}\right]$, the correlation $\sigma^{\mathcal{M}}\left[l_{1}, x_{1} ; l_{2}, x_{2} ; \ldots ; l_{k}, x_{k}\right]$ approaches

$\sigma^{\mathcal{M}}\left[l_{1}, x_{1}\right] \sigma^{\mathcal{M}}\left[l_{2}, x_{2} ; \ldots ; l_{k}, x_{k}\right]$ exponentially fast in the distance measure between $x_{1}$ and $\mathcal{R}\left[x_{2}, \ldots, x_{k}\right]$ along $l_{1}$.

Lemma 4 With notation as above, if $l_{1}$ hits $\mathcal{R}\left[x_{2}, \ldots, x_{k}\right]$ then we have

$$
\begin{gathered}
\sigma^{\mathcal{M}}\left[l_{1}, x_{1} ; l_{2}, x_{2} ; \ldots ; l_{k}, x_{k}\right]=\sigma^{\mathcal{M}}\left[l_{1}, x_{1}\right] \sigma^{\mathcal{M}}\left[l_{2}, x_{2} ; \ldots ; l_{k}, x_{k}\right] \\
\left(1+O\left(\mathrm{e}^{-\mathcal{M}\left(\left[\left[l_{1}\left[x_{1} \leftrightarrow \mathcal{R}\left[x_{2}, \ldots, x_{k}\right]\right]\right]\right]\right)}\right)\right)
\end{gathered}
$$

where $l_{1}\left[x_{1} \leftrightarrow \mathcal{R}\left[x_{2}, \ldots, x_{k}\right]\right]$ is the segment of $l_{1}$ between $x_{1}$ and $\mathcal{R}\left[x_{2}, \ldots, x_{k}\right]$.

Proof Our proof relies on estimating the conditional probability of the event

$$
\mathcal{E}\left[d l_{1}, x_{1}\right]:=\left\{\exists_{e \in \operatorname{Edges}\left(\mathcal{A}^{\mathcal{M}}\right)} x_{1} \in e, l[e] \in d l_{1}\right\}
$$

given the event

$$
\mathcal{E}\left[d l_{2}, x_{2} ; \ldots ; d l_{k}, x_{k}\right]:=\left\{\forall_{i=2}^{k} \exists_{e \in \operatorname{Edges}\left(\mathcal{A}^{\mathcal{M}}\right)} x_{i} \in e, l[e] \in d l_{i}\right\}
$$

We shall show that

$$
\mathbb{P}\left(\mathcal{E}\left[d l_{1}, x_{1}\right] \mid \mathcal{E}\left[d l_{2}, x_{2} ; \ldots ; d l_{k}, x_{k}\right]\right)=\mathcal{M}\left(d l_{1}\right)\left(1+O\left(\mathrm{e}^{-\mathcal{M}\left(\left[\left[l_{1}\left[x_{1} \leftrightarrow \mathcal{R}\left[x_{2}, \ldots, x_{k}\right]\right]\right]\right]\right)}\right)\right)
$$


which will complete the proof in view of (10) [recall that $\left.x_{i} \in l_{i}, i=1, \ldots, k\right]$ and Theorem 4. To proceed, denote by $\tilde{\mathcal{A}}^{\mathcal{M}}$ a rectangular field coinciding in law with $\mathcal{A}^{\mathcal{M}}$ conditioned on the event $\mathcal{E}\left[d l_{2}, x_{2} ; \ldots ; d l_{k}, x_{k}\right]$. Note that since $l_{1}$ crosses $\mathcal{R}\left[x_{2}, \ldots, x_{k}\right]$, it has to be a vertical line. Construct the following directional decision rule $\phi(\cdot, \cdot)$ for the defective dynamics:

- Whenever turning into a horisontal direction, move away from $l_{1}$, that is to say rightwards if the turning point lies to the right from $l_{1}$ and leftwards if the turning point lies to the left of $l_{1}$. When the turning point lies on $l_{1}$, move rightwards.

- Whenever turning into a vertical direction, move upwards.

Moreover, let $D:=\mathcal{R}\left[x_{1}, x_{2}, \ldots, x_{k}\right]$, that is to say $D$ is the smallest rectangle with sides parallel to coordinate axes, spanned by $x_{1}, \ldots, x_{k}$. Furthermore, set $A_{L}$ to be the family of all vertical lines in $[[D]]$ and for each $l \in A_{L}=[[D]]$ let $\mathbb{A}^{*}(l)$ be the topmost point of $l \cap D$ so that in particular $\mathbb{A}^{*}\left(l_{1}\right)=x_{1}$. With this notation, using Theorem 10 including the reversibility statement there we see that the law of the conditional field $\tilde{\mathcal{A}}_{D}^{\mathcal{M}}$ is invariant and reversible for the following conditional version of the defective disagreement loop dynamics:

- With intensity $\mathcal{M}(d l) d s$ for $l \in A_{L}$ set $\gamma_{s+d s}:=\gamma_{s} \oplus\left(l, \mathbb{A}^{*}(l)\right)$. Should the proposed update fail or result in a configuration violating $\mathcal{E}\left[d l_{2}, x_{2} ; \ldots ; d l_{k}, x_{k}\right]$, keep $\gamma_{s+d s}=\gamma_{s}$.

- For each line $l \in A_{L}$ extending an edge $e$ of $\gamma_{s}$ such that $\mathbb{A}^{*}(l) \in e$, with intensity $d s$ set $\gamma_{s+d s}:=\gamma_{s} \ominus\left(l, \mathbb{A}^{*}(l)\right)$. Should the proposed update fail or result in a configuration violating $\mathcal{E}\left[d l_{2}, x_{2} ; \ldots ; d l_{k}, x_{k}\right]$, keep $\gamma_{s+d s}=\gamma_{s}$.

Note that the only difference compared to the DefDL dynamics in Subsection 6.4 is that we discard updates resulting in violation of $\mathcal{E}\left[d l_{2}, x_{2} ; \ldots ; d l_{k}, x_{k}\right]$. Observe also that

- Under the so-defined directional decision rules the disagreement loops arising in the course of the dynamics are always single-branched (the second branch is chopped off by the boundary of $D$ ) and contain no cycles so that the updates in the above dynamics never fail (but note that they can nevertheless be discarded whenever violating $\left.\mathcal{E}\left[d l_{2}, x_{2} ; \ldots ; d l_{k}, x_{k}\right]\right)$.

- The only update of the dynamics which can lead to a transition from a configuration where $\mathcal{E}\left[d l_{1}, x_{1}\right]$ holds to a configuration where it does not hold is $\ominus\left(l_{1}, x_{1}\right)$. Indeed, 
in view of the directional decision rules, even if some disagreement loop annihilates a subsegment of $l_{1}$, this subsegment cannot reach $x_{1}$.

- Likewise, the only update of the dynamics which can lead to a transition from a configuration where $\mathcal{E}\left[d l_{1}, x_{1}\right]$ does not hold to a configuration where it holds is $\oplus\left(l_{1}, x_{1}\right)$.

- For the update $\oplus\left(l_{1}, x_{1}\right)$ the only possibility to cause violation of $\mathcal{E}\left[d l_{2}, x_{2} ; \ldots ; d l_{k}, x_{k}\right]$, and thus to be discarded, is that the initial downwards creation phase growth of the disagreement loop from $x_{1}$ continue with no directional updates along $l_{1}$ until the loop reaches $\mathcal{R}\left[x_{2}, \ldots, x_{k}\right]$. Indeed, if a directional update occured prior to that, the disagreement loop would only unfold upwards and rightwards thereafter, never reaching $\mathcal{R}\left[x_{2}, \ldots, x_{k}\right]$ and consequently not violating $\mathcal{E}\left[d l_{2}, x_{2} ; \ldots ; d l_{k}, x_{k}\right]$. However, the probability that the initial segment of the disagreement loop for $\oplus\left(x_{1}, l_{1}\right)$ extends all the way down to $\mathcal{R}\left[x_{2}, \ldots, x_{k}\right]$ without directional updates is $\exp \left(-\mathcal{M}\left(\left[\left[l_{1}\left[x_{1} \leftrightarrow\right.\right.\right.\right.\right.$ $\left.\left.\left.\left.\left.\mathcal{R}\left[x_{2}, \ldots, x_{k}\right]\right]\right]\right]\right)\right)$ by the definition of the dynamics. Clearly, even if this happens, the violation of $\mathcal{E}\left[d l_{2}, x_{2} ; \ldots ; d l_{k}, x_{k}\right]$ is not guaranteed, but possible.

- Likewise, the update $\ominus\left(l_{1}, x_{1}\right)$, only possible on the event $\mathcal{E}\left[d l_{1}, x_{1}\right]$, may only cause violation of $\mathcal{E}\left[d l_{2}, x_{2} ; \ldots ; d l_{k}, x_{k}\right]$ if the current configuration $\gamma_{s}$ contains a segment along $l_{1}$ joining $x_{1}$ to $\mathcal{R}\left[x_{2}, \ldots, x_{k}\right]$. On the event $\mathcal{E}\left[d l_{1}, x_{1}\right] \cap \mathcal{E}\left[d l_{2}, x_{2} ; \ldots ; d l_{k}, x_{k}\right]$ this only happens with probability $O\left(\exp \left(-\mathcal{M}\left(\left[\left[l_{1}\left[x_{1} \leftrightarrow \mathcal{R}\left[x_{2}, \ldots, x_{k}\right]\right]\right]\right]\right)\right)\right)$ though, in analogy to the previous case.

In view of these observations and by the definition of the dynamics we see that in its course:

- On $\neg \mathcal{E}\left[d l_{1}, x_{1}\right]$, transitions leading to $\mathcal{E}\left[d l_{1}, x_{1}\right]$ occur in s-time with intensity

$$
\mathcal{M}\left(d l_{1}\right) d s\left(1+O\left(\mathrm{e}^{\left.-\mathcal{M}\left(\left[l_{1}\left[x_{1} \leftrightarrow \mathcal{R}\left[x_{2}, \ldots, x_{k}\right]\right]\right]\right]\right)}\right)\right)
$$

- On $\mathcal{E}\left[d l_{1}, x_{1}\right]$, transitions leading to $\neg \mathcal{E}\left[d l_{1}, x_{1}\right]$ occur in s-time with intensity

$$
d s\left(1+O\left(\mathrm{e}^{\left.-\mathcal{M}\left(\left[l_{1}\left[x_{1} \leftrightarrow \mathcal{R}\left[x_{2}, \ldots, x_{k}\right]\right]\right]\right]\right)}\right)\right) .
$$

Consequently, using the detailed balance we see that the probability of $\mathcal{E}\left[d l_{1}, x_{1}\right]$ for $\tilde{\mathcal{A}}^{\mathcal{M}}$, or equivalently the conditional probability of $\mathcal{E}\left[d l_{1}, x_{1}\right]$ given $\mathcal{E}\left[d l_{2}, x_{2} ; \ldots ; d l_{k}, x_{k}\right]$, is

$$
\mathcal{M}\left(d l_{1}\right)\left(1+O\left(\mathrm{e}^{\left.-\mathcal{M}\left(\left[l_{1}\left[x_{1} \leftrightarrow \mathcal{R}\left[x_{2}, \ldots, x_{k}\right]\right]\right]\right]\right)}\right)\right)
$$


as required for (21). This completes the proof of the lemma.

A repetitive use of Lemma 4 readily yields the following approximate factorisation theorem.

Theorem 11 Assume the collection $\left(l_{i}, x_{i}\right)_{i=1}^{k}$ is such that $x_{i} \notin \mathcal{R}\left[x_{i+1}, \ldots, x_{k}\right]$ for all $i$ and let $\delta_{i}:=\mathcal{M}\left[\left[l_{i}\left[x_{i} \leftrightarrow \mathcal{R}\left[x_{i+1}, \ldots, x_{k}\right]\right]\right]\right]$ if $l_{i}$ hits $\mathcal{R}\left[x_{i+1}, \ldots, x_{k}\right]$ and $\delta_{i}:=+\infty$ otherwise. Then

$$
\sigma^{\mathcal{M}}\left[d l_{1}, x_{1} ; \ldots ; d l_{k}, x_{k}\right]=\left(\prod_{i=1}^{k} \mathcal{M}\left(d l_{i}\right)\right) \prod_{i=1}^{k}\left(1+O\left(\exp \left(-\delta_{i}\right)\right)\right) .
$$

\section{Contour birth and death dynamics}

The purpose of the present section is to describe an alternative dynamics which can be used to simulate the empty-boundary field $\mathcal{A}_{D \mid \emptyset}^{\mathcal{M} ; \beta}$ for $\beta \geq 2$. A particular feature of this dynamics is that it yields a perfect sampler, i.e. the sample it outputs comes exactly from the target distribution and not just its approximation. Moreover, for $\beta$ large enough it extends to the whole plane thus allowing for a direct graphical construction (perfect simulation) of the whole plane thermodynamic limit $\mathcal{A}^{\mathcal{M} ; \beta}$. The idea underlying the graphical representation comes from Fernández, Ferrari \& Garcia [7, 8, 9] and in the context of homogeneous polygonal fields it has been developed in Schreiber $[15,16]$. Here we present an extension of the construction to the general non-homogeneous setting. Our presentation splits into several steps.

\subsection{Free contour measure}

Choose an open bounded set $D$ with piecewise smooth boundary and consider the space $\mathcal{C}_{D}$ consisting of all closed polygonal contours in $D$ which do not touch the boundary $\partial D$. For a given finite collection $(l):=\left(l_{1}, \ldots, l_{n}\right)$ of straight lines intersecting $D$ denote by $\mathcal{C}_{D}(l)$ the family of those polygonal contours in $\mathcal{C}_{D}$ which belong to $\Gamma_{D \mid \emptyset}(l):=\Gamma_{D}(l) \cap \Gamma_{D \mid \emptyset}$. We define the so-called free contour measure $\Theta_{D}$ on $\mathcal{C}_{D}$ by putting for $C \subseteq \mathcal{C}_{D}$ measurable, say with respect to the Borel $\sigma$-field generated by the Hausdorff distance topology,

$$
\Theta_{D}(C)=\int_{\operatorname{Fin}(L[D])} \sum_{\theta \in C \cap \mathcal{C}_{D}(l)} \exp \left(-L^{\mathcal{M}}(\theta)\right) d \mathcal{M}^{*}((l))
$$


with Fin $(L[D])$ standing for the for the family of finite collections of lines intersecting $D$ and where $\mathcal{M}^{*}$ is the measure on $\operatorname{Fin}(L[D])$ given by $d \mathcal{M}^{*}\left(\left(l_{1}, \ldots, l_{n}\right)\right):=\prod_{i=1}^{n} \mathcal{M}\left(d l_{i}\right)$. Thus, in other words,

$$
\Theta_{D}(d \theta)=\exp \left(-L^{\mathcal{M}}(\theta)\right) \prod_{e \in \operatorname{Edges}(\theta)} \mathcal{M}(d l[e]) .
$$

For general $\beta \in \mathbb{R}$ we consider the exponential modification $\Theta_{D}^{[\beta]}$ of the free measure $\Theta_{D}$,

$$
\Theta_{D}^{[\beta]}(d \theta):=\exp \left(-[\beta-1] L^{\mathcal{M}}(\theta)\right) \Theta_{D}(d \theta)=\exp \left(-\beta L^{\mathcal{M}}(\theta)\right) \prod_{e \in \operatorname{Edges}(\theta)} \mathcal{M}(d l[e]) .
$$

It is easily seen that the total mass $\Theta_{D}^{[\beta]}\left(\mathcal{C}_{D}\right)$ is always finite for bounded $D$ for all $\beta \in \mathbb{R}$. Indeed, taking into account that $L^{\mathcal{M}}(\theta) \leq \operatorname{card}(\operatorname{Edges}(\theta)) \mathcal{M}([[D]])$ we have in view of (23)

$$
\Theta_{D}^{[\beta]}\left(\mathcal{C}_{D}\right) \leq \sum_{k=0}^{\infty} \frac{1}{k !}[\mathcal{M}([[D]])]^{k} \exp (|\beta| k \mathcal{M}([[D]]))=\exp (\mathcal{M}([[D]]) \exp (|\beta| \mathcal{M}([[D]])))<\infty .
$$

Note that for all $D$ the free contour measures $\Theta_{D}$ as defined in (22) arise as the respective restrictions to $\mathcal{C}_{D}$ of the same measure $\Theta$ on $\mathcal{C}:=\bigcup_{n=1}^{\infty} \mathcal{C}_{(-n, n)^{2}}$, in the sequel referred to as the infinite volume free contour measure. Indeed, this follows easily by the observation that $\Theta_{D_{1}}$ restricted to $\mathcal{C}_{D_{2}}$ coincides with $\Theta_{D_{2}}$ for $D_{2} \subseteq D_{1}$. In the same way we construct the infinite-volume exponentially modified measures $\Theta^{[\beta]}, \beta \in \mathbb{R}$. It turns out that for $\beta \geq 2$ a natural algorithmic random walk representation of $\Theta^{[\beta]}$ can be given. This is done as follows. For each contour $\theta \in \mathcal{C}$ let $\iota[\theta]$ be its leftmost vertex, that is to say its extreme left point minimising the first coordinate, with possible ties broken in an arbitrary measurable way. Next, for $x \in \mathbb{R}^{2}$ denote by $\mathcal{C}_{x}$ the collection of contours $\theta \in \mathcal{C}$ such that $\iota[\theta]=x$. For $\beta \geq 2$ let $\Theta_{x}^{[\beta]}$ be the subprobability measure determined by the following construction of a $\mathcal{C}_{x}$-valued $\Theta_{x}^{[\beta]}$-distributed random element $\theta$

- Simulate a continuous-time random walk $\left(Z_{t}\right)_{t \geq 0}$ gouverned by the following dynamics - choose two random lines $l_{1}, l_{2}$ meeting at $x$ according to the distribution

$$
\frac{d[\mathcal{M} \times \mathcal{M}]\left(\left(l_{1}, l_{2}\right), l_{1} \cap l_{2} \in d x\right)}{2\langle\langle\mathcal{M}\rangle\rangle(d x)},
$$

as in (7),

- put $Z_{0}:=0$ and choose one of two possible initial directions along $l_{1}$ with equal probabilities $1 / 2$, 
- between direction update events specified below move in a constant direction with speed 1 ,

- while moving along the segment $Z_{[t, t+d t]}=\overline{Z_{t} Z_{t+d t}}$, update the movement direction and start moving along a line $l \in\left[\left[Z_{[t, t+d t]}\right]\right]$ with probability $2 \mathcal{M}(d l)$ and with equiprobable choices between the two possible directions along $l$,

- Consider a killed modification $\tilde{Z}_{t}^{[\beta-2]}$ of $Z_{t}$ by killing $Z_{t}$

- with intensity $[\beta-2] \mathcal{M}\left(\left[\left[Z_{[t, t+d t]}\right]\right]\right)$,

- whenever $Z_{t}$ hits its past trajectory,

- Draw an infinite loop-closing half-line $l^{*}$ along $l_{2}$ beginning at $x$ with equiprobable choices of two possible directions,

- If the random walk $\tilde{Z}_{t}^{[\beta-2]}$ hits the loop closing line $l^{*}$ before getting killed, and the self-avoiding contour $\theta_{*}:=\theta_{*}\left[\tilde{Z}^{[\beta-2]} ; l^{*}\right]$ created by $l^{*}$ and the trajectory of $\tilde{Z}_{t}^{[\beta-2]}$ up to the moment of hitting $l^{*}$ satisfies $\iota\left[\theta_{*}\right]=x$, then

- with probability $\exp \left(-\beta \mathcal{M}\left(\left[\left[e^{*}\right]\right]\right)\right)$ output $\theta:=\theta_{*}$, where $e^{*}$ stands for the segment of the loop closing line $l^{*}$ from $x$ to its intersection point with $\tilde{Z}_{t}^{[\beta-2]}$,

- otherwise output $\theta:=\emptyset$.

In all remaining cases set $\theta:=\emptyset$.

Note that outputting $\emptyset$ means failing in the above algorithm, and hence the resulting distribution is a sub-probability rather than probability measure.

The following lemma provides a constructive representation for $\Theta^{[\beta]}, \beta \geq 2$, and is close in spirit to Lemma 5.1 in [16] and Lemma 1 in [12].

Lemma 5 For $\beta \geq 2$ we have

$$
\Theta^{[\beta]}=4 \int_{\mathbb{R}^{2}} \Theta_{x}^{[\beta]}\langle\langle\mathcal{M}\rangle\rangle(d x)
$$


Proof The directed nature of the random walk trajectories as constructed above requires considering for each contour $\theta$ two oriented instances $\theta^{\rightarrow}$ (clockwise) and $\theta^{\leftarrow}$ (anticlockwise). By the construction of $\Theta_{x}^{[\beta]}$ the assertion of the lemma will follow as soon as we show that for each $x \in \mathbb{R}^{2}$ and $\theta \in \mathcal{C}_{x}$ we have

$$
8\langle\langle\mathcal{M}\rangle\rangle(d x) \mathrm{e}^{-\beta L^{\mathcal{M}}\left(e^{*}\right)} \mathbb{P}\left(\tilde{Z}_{t}^{[\beta-2]} \text { reaches } l^{*} \text { and } \theta_{*}\left[\tilde{Z}^{[\beta-2]} ; l^{*}\right] \in d \theta^{\rightarrow}\right)=\Theta^{[\beta]}(d \theta),
$$

where $e^{*}$ stands for the last segment of $\theta^{\rightarrow}$ counting from $x$ as the initial vertex, which is to coincide with the segment of the loop-closing line $l^{*}$ joining to $x$ its intersection point with $\tilde{Z}_{t}^{[\beta-2]}$; whereas $\theta_{*}\left[\tilde{Z}^{[\beta-2]} ; l^{*}\right]$ is the self-avoiding contour created by $l^{*}$ and the trajectory of $\tilde{Z}_{t}^{[\beta-2]}$ up to the moment of hitting $l^{*}$, as denoted in the construction of $\Theta_{x}^{[\beta]}$ above. Indeed, the same relation holds then for $\theta^{\leftarrow}$, so adding versions of (25) for $\theta^{\rightarrow}$ and $\theta^{\leftarrow}$, which amounts to taking into account two possible directions in which the random walk can move along $\theta$, will yield $2 \Theta^{[\beta]}(d \theta)$ on the right hand side, whence the assertion of the lemma will follow.

To establish (25), we observe that the probability element

$$
\mathbb{P}\left(\tilde{Z}_{t}^{[\beta-2]} \text { reaches } l^{*} \text { and } \theta_{*}\left[\tilde{Z}^{[\beta-2]} ; l^{*}\right] \in d \theta^{\rightarrow}\right)
$$

is exactly

$$
\frac{1}{8\langle\langle\mathcal{M}\rangle\rangle(d x)} \exp \left(-\beta L^{\mathcal{M}}\left(\theta \backslash e^{*}\right)\right) \prod_{i=1}^{k} \mathcal{M}\left(d l\left[e_{k}\right]\right)
$$

where $e_{1}, \ldots, e_{k}$ are all segments of $\theta$ including $e^{*}=e_{k}$, while $l\left[e_{i}\right]$ stands for the straight line determined by $e_{i}$. Indeed,

- the prefactor $[8\langle\langle\mathcal{M}\rangle\rangle(d x)]^{-1}=\left[4[\mathcal{M} \times \mathcal{M}]\left(\left\{\left(l_{1}, l_{2}\right) \mid l_{1} \cap l_{2} \in d x\right\}\right)\right]^{-1}$ times the product $\mathcal{M}\left(d l\left[e_{1}\right]\right) \mathcal{M}\left(d l\left[e_{k}\right]\right)$ comes from the choice of the lines $l_{1}$ and $l_{2}$ containing respectively the initial segment of $\theta^{\rightarrow}$ (counting from $x$ ) and the half-line $l^{*}$, as well as from the choice between two equiprobable directions on each of these lines,

- for each of the remaining segments $e_{i}, i=2, \ldots, k-1$, the factor $\mathcal{M}\left(d l\left[e_{i}\right]\right)$ comes from the directional update of the random walk $Z_{t}$, with $2 \mathcal{M}\left(d l\left[e_{i}\right]\right)$ due to the choice of the line and $1 / 2$ due to the choice between two equiprobable directions along this line, 
- finally, $\exp \left(-[\beta-2] L^{\mathcal{M}}\left(\theta \backslash e^{*}\right)\right)$ comes from killing in the course of the random walk whereas extra $\exp \left(-2 L^{\mathcal{M}}\left(\theta \backslash e^{*}\right)\right)$ is due to the absence of directional updates along the segments of the walk, which yields $\exp \left(-\beta L^{\mathcal{M}}\left(\theta \backslash e^{*}\right)\right)$ when put together.

Recalling (23) we see that the expression in (26) coincides with

$$
\frac{1}{8\langle\langle\mathcal{M}\rangle\rangle(d x)} \exp \left(\beta L^{\mathcal{M}}\left(e^{*}\right)\right) \Theta^{[\beta]}(d \theta),
$$

which yields the required relation (25). The proof is complete.

To proceed, assume that the activity measure $\mathcal{M}$ admits a homogeneous upper and lower bound, that is to say

$$
C_{-} \mu \leq \mathcal{M} \leq C_{+} \mu
$$

for some $0<C_{-} \leq C_{+}<\infty$ and with $\mu$ standing for the standard isometry-invariant Haar-Lebesgue measure on the space $\left[\left[\mathbb{R}^{2}\right]\right]$ of straight lines in $\mathbb{R}^{2}$. Recall that one possible construction of $\mu$ goes by identifying a straight line $l$ with the pair $(\phi, \rho) \in[0, \pi) \times \mathbb{R}$, where $(\rho \sin (\phi), \rho \cos (\phi))$ is the vector orthogonal to $l$, and joining it to the origin, and then by endowing the parameter space $[0, \pi) \times \mathbb{R}$ with the usual Lebesgue measure. Then, for each linear segment $e$ we have by standard integral geometry

$$
2 C_{-} \ell(e) \leq \mathcal{M}([[e]]) \leq 2 C_{+} \ell(e)
$$

because $\mu([[e]])=2 \ell(e)$. Likewise, since $\langle\langle\mu\rangle\rangle(d x)=\pi d x$ as easily checked by straightforward integration,

$$
C_{-} \pi d x \leq\langle\langle\mathcal{M}\rangle\rangle(d x) \leq C_{+} \pi d x
$$

Consequently, we conclude from Lemma 5 that under $\Theta^{[\beta]}, \beta \geq 2$, the contour size exhibits exponentially decaying tails, which is a non-homogeneous counterpart of Lemma 1 in [15].

Lemma 6 Under the assumption (27), for $\beta \geq 2$ we have

$$
\Theta^{[\beta]}(\{\theta ; d x \cap \operatorname{Vertices}(\theta) \neq \emptyset, \ell(\theta)>R\}) \leq 4 C_{+} \pi \exp \left(-2 C_{-}[\beta-2] R\right) d x,
$$

where the event $\{d x \cap \operatorname{Vertices}(\theta) \neq \emptyset\}$ is to be understood that a vertex of $\theta$ falls into $d x$. Moreover, there exists a constant $\varepsilon>0$ such that, for $\beta \geq 2$,

$$
\Theta^{[\beta]}(\{\theta ; \mathbf{0} \in \operatorname{Int} \theta, \ell(\theta)>R\}) \leq \exp \left(-2 C_{-}[\beta-2+\varepsilon] R+o(R)\right),
$$

with Int $\theta$ standing for the region enclosed by the contour $\theta$. 
Proof The first assertion follows by the construction of the random walk $Z_{t}$ where, in view of (28), the killing intensity is at least $2 C_{-}$times the length element covered under the present assumptions, whereas the extreme left vertices of contours have their intensity bounded by $4\langle\langle\mathcal{M}\rangle\rangle(d x) \leq 4 C_{+} \pi$ in view of the integral formula in Theorem 5 and of $(29)$. To get the second assertion observe in addition that during each unit time of its evolution the random walk $Z_{t}$ has some uniformly non-zero chance of hitting its past trajectory, see also the proof of Lemma 1 in [15].

\subsection{Polymer representation}

To proceed we let $\mathcal{P}_{\Theta_{D}^{[\beta]}}$ be the Poisson point process in $\mathcal{C}_{D}$ with intensity measure $\Theta_{D}^{[\beta]}$. It follows then directly by (23) in view of (2) and (3) that, for all $\beta \in \mathbb{R}, \mathcal{A}_{D \mid \emptyset}^{\mathcal{M} ; \beta}$ coincides in distribution with the union of contours in $\mathcal{P}_{\Theta_{D}^{[\beta]}}$ conditioned on the event that they are disjoint so that

$$
\mathcal{L}\left(\mathcal{A}_{D \mid \emptyset}^{\mathcal{M} ; \beta}\right)=\mathcal{L}\left(\bigcup_{\theta \in \mathcal{P}_{\Theta_{D}^{[\beta]}}} \theta \mid \forall_{\theta, \theta^{\prime} \in \mathcal{P}_{\Theta_{D}}^{[\beta]}} \theta \neq \theta^{\prime} \Rightarrow \theta \cap \theta^{\prime}=\emptyset\right)
$$

where the conditioning makes sense because $\Theta_{D}^{[\beta]}\left(\mathcal{C}_{D}\right)$ is finite as shown in $(24)$ above, see also [15], Section 2.2. In particular, in analogy to Subsection 2.2 and Theorem 2 ibidem, the law of $\mathcal{A}_{D \mid \emptyset}^{\mathcal{M} ; \beta}$ is invariant and reversible with respect to the following contour birth and death dynamics $\left(\gamma_{s}^{D}\right)$ on $\Gamma_{D}$.

$(\mathbf{C}: \operatorname{birth}[\beta])$ With intensity $\Theta_{D}^{[\beta]}(d \theta) d s$ do

- Choose a new contour $\theta$,

- If $\theta \cap \gamma_{s}^{D}=\emptyset$, accept $\theta$ and set $\gamma_{s+d s}^{D}:=\gamma_{s}^{D} \cup \theta$,

- Otherwise reject $\theta$ and keep $\gamma_{s+d s}^{D}:=\gamma_{s}^{D}$,

$(\mathbf{C}: \operatorname{death}[\beta])$ With intensity $1 \cdot d s$ for each contour $\theta \in \gamma_{s}^{D}$ remove $\theta$ from $\gamma_{s}^{D}$ setting $\gamma_{s}^{D}:=\gamma_{s}^{D} \backslash \theta$

Moreover, $\mathcal{L}\left(\mathcal{A}_{D}^{\mathcal{M} ; \beta}\right)$ is the unique invariant distribution of the above dynamics, see Theorem 2 in [15]. Clearly, both the representation (30) and the above dynamics can be regarded as a kind of polymer representation for $\mathcal{A}_{D}^{\mathcal{M} ; \beta}$. 
Unlike the results for free contour measures, the polymer representation is valid for all values of $\beta$. In this context it is helpful to note that, by (30), the probability $\mathbb{P}\left(\mathcal{A}_{D \mid \emptyset}^{\mathcal{M} ; \beta}=\emptyset\right)$ is not smaller than $\exp \left(-\Theta_{D}^{[\beta]}\left(\mathcal{C}_{D}\right)\right)$. On the other hand, comparing with (2) shows that $\mathbb{P}\left(\mathcal{A}_{D \mid \emptyset}^{\mathcal{M} ; \beta}=\emptyset\right)$ is $\left[\mathcal{Z}_{D \mid \emptyset}^{\mathcal{M} ; \beta}\right]^{-1}$ where, recall, $\mathcal{Z}_{D \mid \emptyset}^{\mathcal{M} ; \beta}$ is the partition function for $\mathcal{A}_{D \mid \emptyset}^{\mathcal{M}}$. Thus, by $(24)$,

$$
\mathcal{Z}_{D \mid \emptyset}^{\mathcal{M} ; \beta} \leq \exp \left(\Theta^{[\beta]}\left(\mathcal{C}_{D}\right)\right)<\infty
$$

Likewise,

$$
\mathcal{Z}_{D}^{\mathcal{M} ; \beta}<\infty
$$

which can be proven along the same lines, see also the proof of Corollary 2 in [15].

\subsection{Graphical representation}

These observations place us within the framework of the general contour birth and death graphical construction as developed by Fernández, Ferrari \& Garcia $[7,8,9]$ and as sketched below, see ibidem and [15] for further details. Choose $\beta$ large enough, to be specified below. Define $\mathcal{F}(\mathcal{C})$ to be the space of countable and locally finite collections of contours from $\mathcal{C}$, with the local finiteness requirement meaning that at most a finite number of contours can hit a bounded subset of $\mathbb{R}^{2}$. On the time-space $\mathbb{R} \times \mathcal{F}(\mathcal{C})$ we construct the time-stationary free contour birth and death process $\left(\varrho_{s}\right)_{s \in \mathbb{R}}$ with the birth intensity measure given by $\Theta^{[\beta]}$ and with the death intensity 1 . Note that free means here that every new-born contour is accepted regardless of whether it hits the union of already existing contours or not, moreover we admit negative time here, letting $s$ range through $\mathbb{R}$ rather than just $\mathbb{R}_{+}$. Observe also that we need the birth measure $\Theta^{[\beta]}$ to be finite on the sets $\{\theta \in \mathcal{C} \mid \theta \cap A \neq$ $\emptyset\}$ for all bounded Borel $A \subseteq \mathbb{R}^{2}$ in order to have the process $\left(\varrho_{s}\right)_{s \in \mathbb{R}}$ well defined on $\mathbb{R} \times \mathcal{F}(\mathcal{C})$. By Lemma 6 this is ensured whenever $\beta \geq 2$. To proceed, for the free process $\left(\varrho_{s}\right)_{s \in \mathbb{R}}$ we perform the following trimming procedure. We place a directed connection from each time-space instance of a contour showing up in $\left(\varrho_{s}\right)_{s \in \mathbb{R}}$ and denoted by $\theta \times\left[s_{0}, s_{1}\right)$, with $\theta$ standing for the contour and $\left[s_{0}, s_{1}\right)$ for its lifespan, to all time-space contour instances $\theta^{\prime} \times\left[s_{0}^{\prime}, s_{1}^{\prime}\right)$ with $\theta^{\prime} \cap \theta \neq \emptyset, s_{0}^{\prime} \leq s_{0}$ and $s_{1}^{\prime}>s_{0}$. In other words, we connect $\theta \times\left[s_{0}, s_{1}\right)$ to its ancestors understood as those contour instances which may have affected the acceptance status of $\theta \times\left[s_{0}, s_{1}\right)$ in the constrained contour birth and death dynamics (C) as discussed above. These directed connections give rise to directed ancestor chains 
of time-space contour instances, following Fernandez, Ferrari \& Garcia [9] the union of all ancestor chains stemming from a given contour instance $\theta^{*}=\theta \times\left[s_{0}, s_{1}\right)$, including the instance itself, is referred to as its clan of ancestors and is denoted by $\operatorname{An}\left(\theta^{*}\right)$. More generally, for a bounded region $U$ in the plane we write $\operatorname{An}_{s}(U)$ for the union of ancestor clans of all contour instances $\theta \times\left[s_{0}, s_{1}\right)$ with $\theta \cap U \neq \emptyset$ and $s \in\left[s_{0}, s_{1}\right)$. Lemma 6 allows us to apply the technique of domination by sub-critical branching processes, developed in $[7,8,9]$, in order to conclude that there exists $\beta_{g}$ such that for each $\beta>\beta_{g}$ there exists $c:=c(\beta)>0$ such that

$$
\mathbb{P}\left(\operatorname{diam} \operatorname{An}_{s}\left(\mathbb{B}_{2}(x, 1)\right)>R\right) \leq \exp (-c R), s \in \mathbb{R}, x \in \mathbb{R}^{2},
$$

with $\mathbb{B}_{2}(x, 1)$ standing for the ball of radius 1 in $\mathbb{R}^{2}$ centred at $x$. In the sequel we shall always assume that $\beta>\beta_{g}$, that is to say that $\beta$ is in the validity region of the graphical construction. We see that for $\beta>\beta_{g}$ all the ancestor clans are a.s. finite and we can uniquely determine the acceptance status of all their members: contour instances with no ancestors are a.s. accepted, which automatically and uniquely determines the acceptance status of all the remaining members of the clan by recursive application of the inter-contour exclusion rule. In this case, discarding the unaccepted contour instances leaves us with a time-space representation of a time-stationary evolution $\left(\gamma_{s}\right)_{s \in \mathbb{R}}$ on $\mathcal{F}(\mathcal{C})$, which is easily checked to evolve according to the whole-plane version of the dynamics (C) above. In full analogy with Section 4 and Theorem 4 of [15] we see that for all $s \in \mathbb{R}$ the polygonal field $\gamma_{s}$ coincides in distribution with the thermodynamic limit (see Section 3 ibidem) for $\mathcal{A}^{\mathcal{M} ; \beta}$ without infinite contours, which is unique (see Corollary 4 ibidem). For definiteness we put $\mathcal{A}^{\mathcal{M} ; \beta}:=\gamma_{0}$. It should be also observed that for each $s \in \mathbb{R}$ the free field $\varrho_{s}$ coincides in distribution with the Poisson contour process $\mathcal{P}_{\Theta^{[\beta]}}$. Since almost surely we have $\gamma_{s} \subseteq \varrho_{s}$, we get the stochastic domination of the contour ensemble $\mathcal{A}^{\mathcal{M} ; \beta}$ by $\mathcal{P}_{\Theta[\beta]}$. Moreover, using the exponential decay relation (33), in full analogy with Theorem 4 (iv,v) we readily establish the following $\beta$-mixing statement for $\mathcal{A}^{\mathcal{M} ; \beta}$.

Corollary 5 For $\beta>\beta_{g}$ there exists $c>0$ such that for each $A, B \subseteq \mathbb{R}^{2}$ the law of the restriction $\mathcal{A}_{\mid A \cup B}^{\mathcal{M} ; \beta}$ of the thermodynamic limit $\mathcal{A}^{\mathcal{M} ; \beta}$ to $A \cup B$ differs in variational distance from the product of the laws of the respective restrictions $\mathcal{A}_{\mid A}^{\mathcal{M} ; \beta}, \mathcal{A}_{\mid B}^{\mathcal{M} ; \beta}$ by at most $O(\operatorname{cover}(A) \operatorname{cover}(B) \exp (-c \operatorname{dist}(A, B)))$, where $\operatorname{cover}(X)$ stands for the minimum number of unit balls covering $X \subseteq \mathbb{R}^{2}$ whereas $\operatorname{dist}(A, B):=\inf _{x \in A, y \in B} \operatorname{dist}(x, y)$. 
Indeed, this is easily checked by noting that the considered variational distance is bounded above by the probability that either $\operatorname{An}_{0}(A)$ extends further than $\operatorname{dist}(A, B) / 2$ away from $A$ or $\operatorname{An}_{0}(B)$ extends further than $\operatorname{dist}(A, B) / 2$ away from $B$ which happens with probability at most $O(\operatorname{cover}(A) \operatorname{cover}(B) \exp (-c \operatorname{dist}(A, B)))$ as required. Furthermore, in analogy to the discussion following Corollary 4 in [15] we can use the stochastic domination of $\mathcal{A}^{\mathcal{M} ; \beta}$ by $\mathcal{P}_{\Theta[\beta]}$ and apply Lemma 6 to conclude that

Corollary 6 With probability 1 there is only finite contour nesting in $\mathcal{A}^{\mathcal{M} ; \beta}$, that is to say no infinite chains of nested contours are present.

Indeed, this comes from the fact that the expected number of contours surrounding a given point is always finite by Lemma 6 . In addition, for $\beta$ large enough this expected number can be made arbitrarily small, which clearly guarantees the presence of long-range point-topoint correlations and spontaneous magnetisation with contours interpreted as separating phases of different signs, as follows by a standard Peierls-type argument.

We also consider finite-volume versions of the above graphical construction, replacing the infinite-volume birth intensity measure $\Theta^{[\beta]}$ with its finite-volume counterparts $\Theta_{D}^{[\beta]}$ for bounded and open $D$ with piecewise smooth boundary. Clearly, the graphical construction yields then a version of the finite-volume contour birth and death evolution $(\mathbf{C})$. For each $D$ denote by $\left(\gamma_{s}^{D}\right)_{s \in \mathbb{R}}$ the resulting finite-volume time-stationary process on the space $\mathcal{F}\left(\mathcal{C}_{D}\right)$ of finite contour configurations in $D$ and write $\left(\varrho_{s}^{D}\right)_{s \in \mathbb{R}}$ for the corresponding free process. It follows as in Theorem 2 in [15] that $\gamma_{s}^{D}$ coincides in distribution with $\mathcal{A}_{D}^{\mathcal{M} ; \beta}$ for each $s \in \mathbb{R}$. Likewise, $\varrho_{s}^{D}$ coincides in distribution with $\mathcal{P}_{\Theta_{D}^{[\beta]}}$.

By representing the measures $\Theta_{D}^{[\beta]}$ as the corresponding restrictions of $\Theta^{[\beta]}$ we obtain a natural coupling of all the processes $\gamma_{s}^{D}, \varrho_{s}^{D}, \gamma_{s}$ and $\varrho_{s}$ on a common probability space. We shall also consider $\mathcal{A}_{D}^{\mathcal{M} ; \beta}$ coupled on the same probability space by putting $\mathcal{A}_{D}^{\mathcal{M} ; \beta}=\gamma_{0}^{D}$. Under this coupling we have the following corollary stating exponential convergence of $\mathcal{A}_{D}^{\mathcal{M} ; \beta}$ to $\mathcal{A}^{\mathcal{M} ; \beta}$ as $D \uparrow \mathbb{R}^{2}$.

Corollary 7 Assume that $\beta$ is within the validity regime of the contour birth and death graphical construction. Then for $x \in D$ such that $B(x, 1) \subseteq D$ we have

$$
\mathbb{P}\left(\left[\mathcal{A}^{\mathcal{M} ; \beta}\right]_{\mathbb{B}_{2}(x, 1)} \neq\left[\mathcal{A}_{D}^{\mathcal{M} ; \beta}\right]_{\mid \mathbb{B}_{2}(x, 1)}\right) \leq \exp (-c[\operatorname{dist}(x, \partial D)-1]),
$$

with $c$ as in (33) and with $\mid$ standing for the restriction operator. 
Indeed, this follows easily by (33) upon noting that the event $\left\{\left[\mathcal{A}^{\mathcal{M} ; \beta}\right]_{\mid \mathbb{B}_{2}(x, 1)} \neq\left[\mathcal{A}_{D}^{\mathcal{M} ; \beta}\right]_{\mid \mathbb{B}_{2}(x, 1)}\right\}$ only occurs when $\operatorname{An}_{0}\left(\mathbb{B}_{2}(x, 1)\right) \nsubseteq D$.

\section{References}

[1] Arak, T. (1982) On Markovian random fields with finite number of values, 4th USSR-Japan symposium on probability theory and mathematical statistics, Abstracts of Communications, Tbilisi.

[2] Arak, T., Surgailis, D. (1989) Markov Fields with Polygonal Realisations, Probab. Th. Rel. Fields 80, 543-579.

[3] Arak, T., Surgailis, D. (1991) Consistent polygonal fields, Probab. Th. Rel. Fields 89, 319-346.

[4] Arak, T., Clifford, P., Surgailis, D. (1993) Point-based polygonal models for random graphs, Adv. Appl. Probab. 25, 348-372.

[5] Arias-Castro, E. (2007) Interpolation of random hyperplanes, Electronic Journal of Probability, 12, 1052-1071.

[6] Clifford, P., Nicholls, G. (1994). A Metropolis sampler for polygonal image reconstruction. available at: http://www.stats.ox.ac.uk/ clifford/papers/met_poly.html

[7] Fernández, R., Ferrari, P., Garcia, N. (1998) Measures on contour, polymer or animal models. A probabilistic approach. Markov Processes and Related Fields 4, 479-497.

[8] Fernández, R., Ferrari, P., Garcia, N. (2001) Loss network representation of Ising contours Ann. Probab. 29, 902-937.

[9] Fernández, R., Ferrari, P., Garcia, N. (2002) Perfect simulation for interacting point processes, loss networks and Ising models. Stoch. Proc. Appl. 102, 63-88.

[10] Kluszczyński, R., Lieshout, M.N.M. van, Schreiber, T. (2005) An algorithm for binary image segmentation using polygonal Markov fields. In: F. Roli and 
S. Vitulano (Eds.), Image Analysis and Processing, Proceedings of the 13th International Conference on Image Analysis and Processing. Lecture Notes in Comput. Sci. 3615, 383-390.

[11] Kluszczyóski, R., Lieshout, M.N.M. van, Schreiber, T. (2007) Image segmentation by polygonal Markov fields. Ann. Inst. Statist. Math., 59, 465-486.

[12] Lieshout, M.N.M. van, Schreiber, T. (2007) Perfect simulation for lengthinteracting polygonal Markov fields in the plane, Scand. Journal of Statistics, 34, $615-625$.

[13] Liggett, T. (1985). Interacting particle systems. Springer-Verlag, New York.

[14] NicholLS, G.K. (2001) Spontaneous magnetisation in the plane, Journal of Statistical Physics, 102, 1229-1251.

[15] Schreiber, T. (2005) Random dynamics and thermodynamic limits for polygonal Markov fields in the plane, Advances in Applied Probability 37, 884-907.

[16] Schreiber, T. (2006) Dobrushin-Kotecký-Shlosman theorem for polygonal Markov fields in the plane, Journal of Statistical Physics, 123, 631-684.

[17] Schreiber, T., Lieshout, M.N.M. van (2008) Disagreement loop and path creation/annihilation algorithms for binary planar Markov fields with applications to image segmentation, submitted

[18] Surgailis, D. (1991) Thermodynamic limit of polygonal models, Acta applicandae mathematicae, 22, 77-102. 